\title{
Aves fósiles de la Península Ibérica, Canarias y Baleares: balance de los estudios realizados
}

Antonio Sánchez Marco | Institut Català de Paleontologia Miquel Crusafont

URL de la contribución <www.iaph.es/revistaph/index.php/revistaph/article/view/4137>

\section{RESUMEN}

Representantes del grupo de las neornitas son las aves más antiguas que se han descubierto en la Península Ibérica, en el Cretácico inferior. Recientemente, se han hallado evidencias de una gastornítida, aves de gran tamaño y no-voladoras, en sedimentos del Cretácico superior. La información sobre las aves del pasado comienza a ser rica con las cuencas sedimentarias que se forman durante el Mioceno. Las primeras indicaciones de caídas sustanciales de la temperatura en el hemisferio norte que acusan las aves tienen lugar hace aproximadamente tres millones de años. Son también las primeras evidencias de migración anual. Ratites procedentes de África se encuentran entre los animales que se instalaron durante el Plioceno inferior en las primeras islas que más tarde conformarán el archipiélago canario.

\section{Palabras clave}

Aves | Baleares | Canarias | Fósiles | Península Ibérica | 


\section{INTRODUCCIÓN}

Las aves que han vivido en el pasado en la Península Ibérica han dejado un rastro fósil muy discontinuo en el tiempo, y lo mismo se puede afirmar para los archipiélagos canario y balear. Las Hoyas es uno de los pocos yacimientos del Cretácico en el mundo en los que se encuentran enantiornitas. El Paleógeno ibérico es en cuanto a las aves un lapso casi vacío. En el Mioceno existen unos pocos yacimientos con una cierta cantidad de restos óseos, pero la mayoría de las localidades de esta época son muy pobres. En el Plioceno el registro de aves es más rico y las muestras de los distintos grupos de aves, más compensadas. Hace unos 3 millones de años tenemos una ornitocenosis con las características que tendrán las ornitofaunas del Cuaternario. Las aves acusan un enfriamiento en el hemisferio norte. Los registros más antiguos en los archipiélagos canario y balear tienen lugar en el Plioceno. En particular, las aves más antiguas de las que se tiene constancia en las Canarias forman parte de los primeros vertebrados terrestres que llegan a las islas orientales y prosperan durante varios cientos de miles de años. Tanto ambos archipiélagos como la Península Ibérica, cuentan con muchas localidades en las que se han preservado fósiles de aves del Pleistoceno y Holoceno, y algunos de estos yacimientos están entre los más ricos del mundo.

\section{SINOPSIS DE LOS RESULTADOS}

\section{Cretácico}

En dos localidades del Cretácico inferior, La Pedrera del Montsec (Lérida) y Las Hoyas (Cuenca), se han descubierto restos fósiles que se han podido asignar a Enantiornithes y también unos pocos restos pertenecientes a una especie de ave de afinidades taxonómicas inciertas a causa de la ausencia de caracteres diagnósticos. Enantiornithes es uno de los grupos más diversificados del Cretácico. No se han hallado descendientes de estas aves después del límite Cretácico/Paleógeno (ZHOU, 2004).

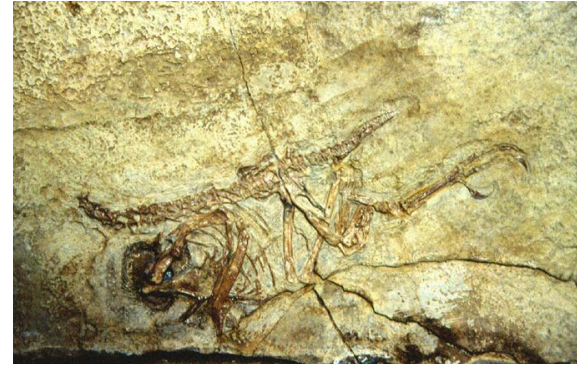

Iberomesornis romerali. Las Hoyas. Cretácico inferior | fuente SANZ; ORTEGA, 2002
En Las Hoyas, de edad Barremiense superior, han aparecido Iberomesornis romerali, Concornis lacustris y Eoalulavis hoyasi. En todos faltan los respectivos cráneos. El primer taxón corresponde a un animal del tamaño de un gorrión. Es destacable un pigostilo muy largo y un coracoides muy robusto. Concornis tiene una talla el doble que la de Iberomesornis (SANZ; ORTEGA, 2002). El fémur y la ulna son notablemente más largos que el conjunto de los huesos de la mano. Posee una fúrcula muy desarrollada, con un hipocleido robusto. En Eoalulavis se encuentra por vez primera el álula, una estructura que consta de unas pocas plumas que el animal mueve con uno de sus dedos del ala para frenar la velocidad y para mejorar la maniobra- 
bilidad en el vuelo. Como también sucede en Concornis, Eoalulavis es un ave con una mano relativamente corta en comparación con los otros huesos del ala.

En el Montsec primeramente se descubrió un pequeño conjunto de restos óseos y partes de plumas, con insuficientes caracteres morfológicos para ser asignados a un grupo particular de las aves que vivieron en el Cretácico, que recibió el nombre de Noguerornis gonzalezi (LACASA RUIZ, 1986; 1989). En el mismo yacimiento apareció posteriormente el cráneo de un individuo juvenil de Enantiornithes. Aun siendo juvenil, este animal era de talla notablemente superior al encontrado previamente (SANZ; CHIAPPE; PÉREZMORENO et ál., 1997).

En el yacimiento de Laño (Burgos), del Cretácico superior, se ha encontrado parte de un sinsacro de un ave de gran tamaño, Gargantuavis philoinos, especie hallada anteriormente en el sur de Francia. Se interpreta que este grupo de aves es resultado de un fenómeno de evolución insular en ausencia de carnívoros de gran talla. Parte de la Península Ibérica y sur de Francia constituían la isla Ibero-Armoricana del archipiélago europeo (BUFFETAUT, 2008; BUFFETAUT; PEREDA-SUBERBIOLA; CORRAL et ál, 2015).

\section{Paleógeno}

Los terreneos paleógenos ibéricos no se distinguen por su riqueza en fósiles de aves. Los únicos conocidos son tres huesos del yacimiento del Eoceno inferior de Silveirinha (Baixo Mondego), atribuidos a la recurvirróstrida Fluviatilavis antunesi (HARRISON, 1983). En varios yacimientos del Oligoceno del norte de la Península -Agramunt, Peralta de la Sal, Liedena y Suria- se han descubierto icnitas producidas por aves. En Tárrega y en Los Barros aparecieron unos escasos fragmentos óseos en muy malas condiciones de conservación (SÁNCHEZ MARCO, 1999a). No se ha trabajado en el estudio de los restos de aves del Paleógeno.

\section{Neógeno peninsular}

El registro de aves del Neógeno es claramente más rico que el de los periodos anteriores, aunque la mayor parte de las localidades fosilíferas proporcionan escasos elementos (SÁNCHEZ MARCO, 1995a, 1995b, 1999a, 2010). No obstante, hay algunos conjuntos de aves que comienzan a ofrecer un cierto conocimiento de las aves de este lapso temporal y apuntan problemáticas de algún interés. Incluso los yacimientos donde se ha hallado mayor variedad taxonómica presentan sesgos claros en la representación de los diversos grupos de aves que se supone que estarían presentes. No hay ninguna localidad o conjunto de localidades de este periodo que ofrezcan muestras equitativas de las ornitocenosis locales. 


\section{Cuenca del Vallés-Penedés}

Es una cuenca sedimentaria que se origina como consecuencia de tensiones tectónicas durante el Neógeno. Sus secuencias sedimentarias comprenden casi todo el Mioceno. Hay diversos sitios donde se han hallado restos de aves (El Fallol-MN3, Can Mas-MN4, Can Poncic-MN9, Hostalets de Pierola indeterminado-MN7+8 y MN9, Viladecavalls-MN10), pero en ninguno de ellos hay una muestra amplia de taxones. Se registran algunos falconiformes, charadriiformes y estrigiformes, pero el de los galliformes es el grupo dominante en esta cuenca. Se han identificado: Miophasianus altus, Miophasianus medius, Palaeortyx brevipes/grivensis, Palaeortyx phasianoides y Palaeortyx gallica (SÁNCHEZ MARCO, 2006a; en prensa). Cada uno de estos taxones tiene una distribución cronológica muy amplia a lo largo del Mioceno europeo. En Hostalets de Pierola se halla Tyto balearica (SÁNCHEZ MARCO, en prensa), afín morfológicamente a la lechuza común actual.

\section{Toril 3-Cuenca de Calatayud}

Toril 3, situado en el municipio de Daroca, es la única localidad conocida con restos de aves de la cuenca de Calatayud. Su fauna corresponde a la biozona MN7+8. Como sucede en la cuenca del Vallés-Penedés, las especies halladas aquí pertenecen casi en su totalidad al grupo de galliformes y son las mismas (Miophasianus altus, Miophasianus medius, Palaeortyx brevipes/grivensis, Palaeortyx phasianoides y Palaeortyx gallica) (SÁNCHEZ MARCO, 2006a; en prensa). En cuanto a las estrigiformes, en Toril 3 aparece la lechuza Tyto sanctialbani (SÁNCHEZ MARCO, 2001).

\section{Cerro de los Batallones}

El conjunto de depósitos paleontológicos del cerro de los Batallones se encuentra en el sur de Madrid. Contiene una amplia muestra de la fauna de vertebrados terrestres de la biozona MN10 (Mioceno superior). Estos yacimientos funcionaron en su momento como oquedades naturales donde quedaban atrapados mamíferos que deambulaban por la zona, lo que constituía un foco de atracción para carnívoros y carroñeros. Los mamíferos son predominantes en estos yacimientos $y$, muy particularmente, los carnívoros (MORALES; POZO; SILVA et ál., 2008). Las especies de aves son asimismo muy abundantes. La mayoría de los elementos óseos avianos que han aparecido son falanges pedales, vértebras y fragmentos de huesos de incierta identificación. Pero un centenar de huesos sí se han podido asignar a especies o géneros. Aunque el estudio sistemático no está concluido, se han distinguido unas doce especies, la mayor parte carroñeras (SÁNCHEZ MARCO, 2017).

En el grupo de las galliformes, Miophasianus medius es la especie de mayor tamaño. Debió de ser parecido a los actuales pavos, y posiblemente con similares requisitos ecológicos. Aparece en otros yacimientos españoles y europeos (MLÍKOVSKÝ, 2002; SÁNCHEZ MARCO, 2006a). También hay algunos 
huesos que corresponderían a aves parecidas a las codornices actuales; bien a Palaeortyx brevipes o a Palaeortyx grivensis (SÁNCHEZ MARCO, en prensa). En los elementos esqueléticos estudiados no se han visto caracteres que permitan separar ambas especies. La distribución cronológica de Palaeortyx brevipes va desde el Paleógeno de Francia (GAILLARD, 1908 -Palaeortyx cayluxensis-; MOURER-CHAUVIRÉ, 1992) hasta Aljezar B (Mioceno superior, España) (CHENEVAL; ADROVER, 1993: Palaeortyx cf. brevipes). Palaeortyx grivensis se encuentra desde el Mioceno medio al superior (MLÍKOVSKÝ, 2002).

En Batallones hay numerosos restos fósiles de un alimoche y de un quebrantahuesos, cuyos representantes actuales son Neophron percnopterus y Gypaetus barbatus, respectivamente. En el Neógeno europeo no se han hallado hasta ahora animales de estas líneas filogenéticas. Sin embargo recientemente se ha descrito en el Mioceno tardío de China Mioneophron longirostris (LI; CLARKE; ZHOU et ál., 2016). En Norteamérica se han descrito seis especies de buitres del género Neophrontops, desde el Mioceno temprano al Pleistoceno (BRODKORB, 1964; FEDUCCIA, 1974; RICH, 1977), que guardan similitudes morfológicas con el alimoche actual (HOWARD, 1966). También aparecen dos buitres; uno con afinidades con los buitres orejudos o negros, Torgos/Aegypius, y el otro de menor tamaño, indistinguible de las especies actuales de Gyps. El registro europeo más antiguo de la subfamilia Aegypiinae es Aegypius tugarinovi, del Plioceno inferior de Moldavia (ZELENKOV; MANEGOLD, 2015). En el Mioceno tardío de China se han descrito varios buitres con afinidades con el grupo Aegypiinae: Mioaegypius gui, en Jiangsu (HOU, 1984), Qiluornis taishanensis (HOU; ZHOU; ZHANG et ál., 2000) y Gansugyps linxiaensis, en Gansu (ZHANG; ZHENG; ZHENG, 2010).

El grupo de falconiformes de la fauna local también comprendía rapaces de tallas medias, como las actuales águilas culebreras y milanos (SÁNCHEZ MARCO, 2017).

\section{Ratites (cáscaras de huevos)}

Las ratites constituyen un grupo monofilético de paleognatas según la mayor parte de los estudios morfológicos y moleculares que se han realizado (HADDRATH; BAKER, 2001; HARSHMAN, 2008). Los representantes actuales de este clado son una especie de avestruz, dos especies de ñandúes, tres de casuarios, una especie de emú y cinco de kiwis. Su registro fósil abarca desde el Paleógeno (MAYR, 2009; CENIZO, 2012), y en el Neógeno hay evidencias de su presencia en casi todo el mundo. Han perdido la capacidad de vuelo y todas, a excepción del kiwi, son corredoras y de talla grande. A pesar del considerable tamaño de las ratites y de que sus huesos son macizos, las cáscaras de huevos son considerablemente más frecuentes que los elementos del esqueleto. En terrenos neógenos de Europa y Asia se hallan cáscaras cuyos poros de intercambio gaseoso en la superficie exterior forman
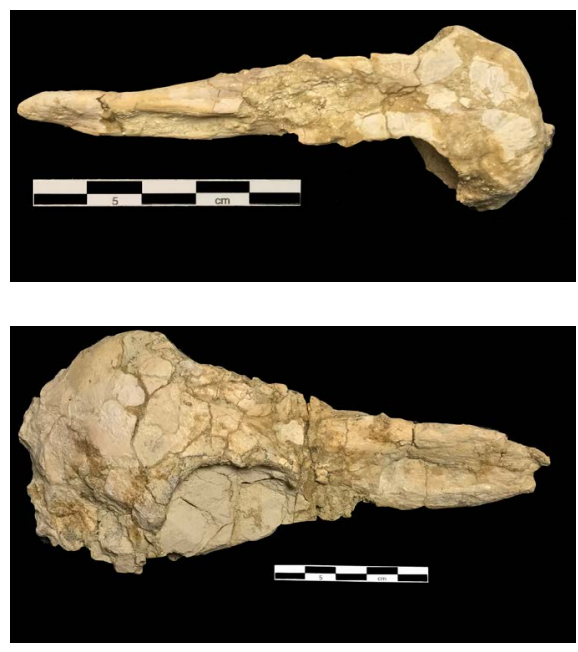

Arriba, cráneo de alimoche; abajo, cráneo de buitre de gran tamaño. Batallones. Mioceno superior | fotos A. Sánchez Marco (2018) 
patrones similares tanto al del actual avestruz (Struthio) como al de las extinguidas aves elefante (Aepyornis) de Madagascar, y estos dos diseños también se encuentran ampliamente distribuidos por África (BOEV; SPASSOV, 2009; PATNAIK; SAHNI; CAMERON et ál., 2009; WANG; HU; WANG, 2012).

Se han encontrado cáscaras de huevo de tipo Aepyornis en dos yacimientos del Mioceno superior de la cuenca del bajo Segura, en Torrellano (BRAVO; YÉBENES; MARTÍN, 2009) y en Crevillente 2 (datos inéditos). EI primero está en el piso Messiniense (BRAVO; YÉBENES; MARTíN, 2009) y el segundo en el Turoliense inferior (MONTOYA, 1997). Mein y Dauphin (1995) reportan la presencia del mismo tipo de cáscaras en la localidad de La Gloria 4 (Plioceno inferior, Fosa de Teruel). También son del Plioceno inferior los hallazgos del norte de Lanzarote, que se tratan más adelante. Tanto en Crevillente 2 como en La Gloria 4 han aparecido huesos fósiles de diversos animales, aves incluidas (SÁNCHEZ MARCO, 1999a; datos inéditos para Crevillente 2), pero no hay evidencias de huesos de ratites.

\section{Plioceno}

Durante el Plioceno tiene lugar un cambio profundo en la composición taxonómica de las ornitocenosis. Desaparecen los géneros y especies que han existido durante el Mioceno y son reemplazadas por otras. Hacia el final del Plioceno van apareciendo las especies que caracterizarán las faunas propias del Pleistoceno y del Holoceno. Elementos esqueléticos procedentes del yacimiento de Layna (Soria, Plioceno inferior) han servido para describir un otídido (avutarda), Chlamydotis mesetaria (SÁNCHEZ MARCO, 1990), y una codorniz de talla grande, Megalocoturnix cordoni (SÁNCHEZ MARCO, 2009). También se encuentran aquí restos de la lechuza Tyto balearica (MOURER-CHAUVIRÉ; SÁNCHEZ MARCO, 1988; SÁNCHEZ MARCO, 2001). Ninguna de estas especies vive en la actualidad, pero dos de los géneros, Chlamydotis y Tyto, sí forman parte de las faunas cuaternarias.

En el yacimiento de Las Higueruelas, que se forma durante el Plioceno superior, en un sistema de antiguas lagunas, en el interior de un cono volcánico, por vez primera encontramos representantes de los diversos grupos de aves que componían la ornitofauna local (SÁNCHEZ MARCO, 2005; 2009): Podiceps auritus, Podiceps nigricollis, Ardea cinerea, Nycticorax nycticorax, Ixobrychus minutus, Plegadis falcinellus, Cygnus cf. cygnus, cf. Anser sp., Tadorna sp., Marmaronetta angustirostris, Aythya sp., Aythya marila, Mergus albellus, Oxyura leucocephala, Palaeocryptonyx novaki, Alectoris sp./Perdix sp., Crex crex, Actitis hypoleucos, Pterocles alchata, Columba sp., Anthus pratensis, cf. Turdus sp., Emberiza citrinella, Passer sp., Corvus monedula y Corvus cf. antecorax.

Bonadonna yVilla (1984) obtuvieron una fecha de 3,5 Ma (por datación con el método K/Ar) para este yacimiento, y se incluye en la biozona MN16 según su 
composición faunística (BRUIJN; DAAMS; DAXNER-HÖCK et ál., 1992). No obstante hay varias razones para considerar que es el primer conjunto ornítico típico del Cuaternario (SÁNCHEZ MARCO, 2005). Por primera vez aparecen especies invernantes. Es decir, encontramos las primeras evidencias del fenómeno de la migración anual en las aves. Todas las especies, excepto una, pertenecen al elenco de taxones que hay durante el Pleistoceno y Holoceno.

Además, este conjunto faunístico tiene unas implicaciones inesperadas desde un punto de vista paleoclimático porque las especies migradoras evidencian condiciones climáticas bastante frías en latitudes altas del continente europeo, que solo se registran en el sur de Europa durante el Pleistoceno en coincidencia con las fases de avance del casquete polar norte. Quizá el conjunto de aves de Las Higueruelas es la primera evidencia biológica y contundente del enfriamiento del hemisferio norte que comienza hacia los 3,1 Ma (RAYMO; RUDDIMAN; CLEMENT, 1986; RAYMO, 1991).

\section{Pleistoceno peninsular}

Un rasgo característico del Pleistoceno es que las especies que encontramos son las mismas que hay en la actualidad, con escasas excepciones que se registran al comienzo de esta época. En cuanto a los conjuntos orníticos -es decir, entendiendo que las asociaciones fósiles son muestras de las paleornitocenosis locales-, desde los yacimientos más antiguos se evidencia que la Península Ibérica estuvo dividida en las dos regiones bioclimáticas actuales (mediterránea y eurosiberiana o atlántica), si bien los límites y las extensiones de ambas fluctuaron a lo largo de toda esta época. Los yacimientos del Pleistoceno son tan numerosos, en particular, los de las fases finales, que en este trabajo solo se han podido escoger unos pocos. Incluso se han quedado fuera algunos conjuntos muy interesantes.

\section{Región eurosiberiana o atlántica}

El conjunto de yacimientos de Atapuerca, la cueva de El Castillo y la cueva de Santa Catalina han proporcionado las asociaciones orníticas más ricas y diversas de esta región. Las aves de estos yacimientos, con sus niveles de diversas cronologías, señalan la existencia de una gama amplia de hábitats.

Las tablas 1 y 2 muestran la mayor parte de las especies que han aparecido en algunos de los niveles de los yacimientos de la trinchera de Atapuerca.

Las características ambientales y climáticas que se desprenden al analizar las asociaciones de aves de Atapuerca se han tratado anteriormente (SÁNCHEZ MARCO, 1999b; 1999c; MADE; AGUIRRE; BASTIR et ál., 2003). Quizá se deba destacar que las aves de todos los niveles son propias de climas templa- 


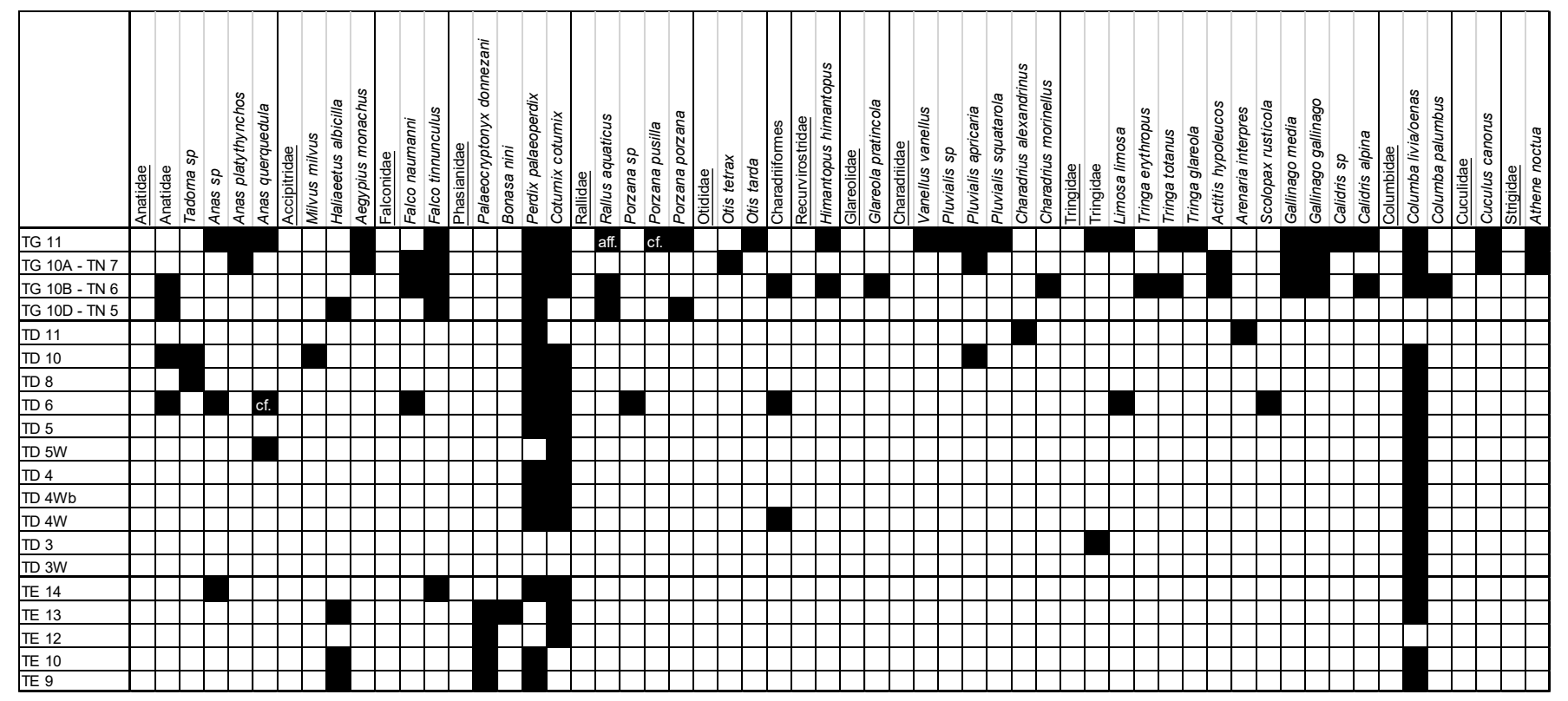

Tabla 1. Distribución por niveles de las aves no passeriformes del conjunto de yacimientos de Atapuerca | fuente MADE; AGUIRRE; BASTIR et ál., 2003; SÁNCHEZ MARCO, 2009

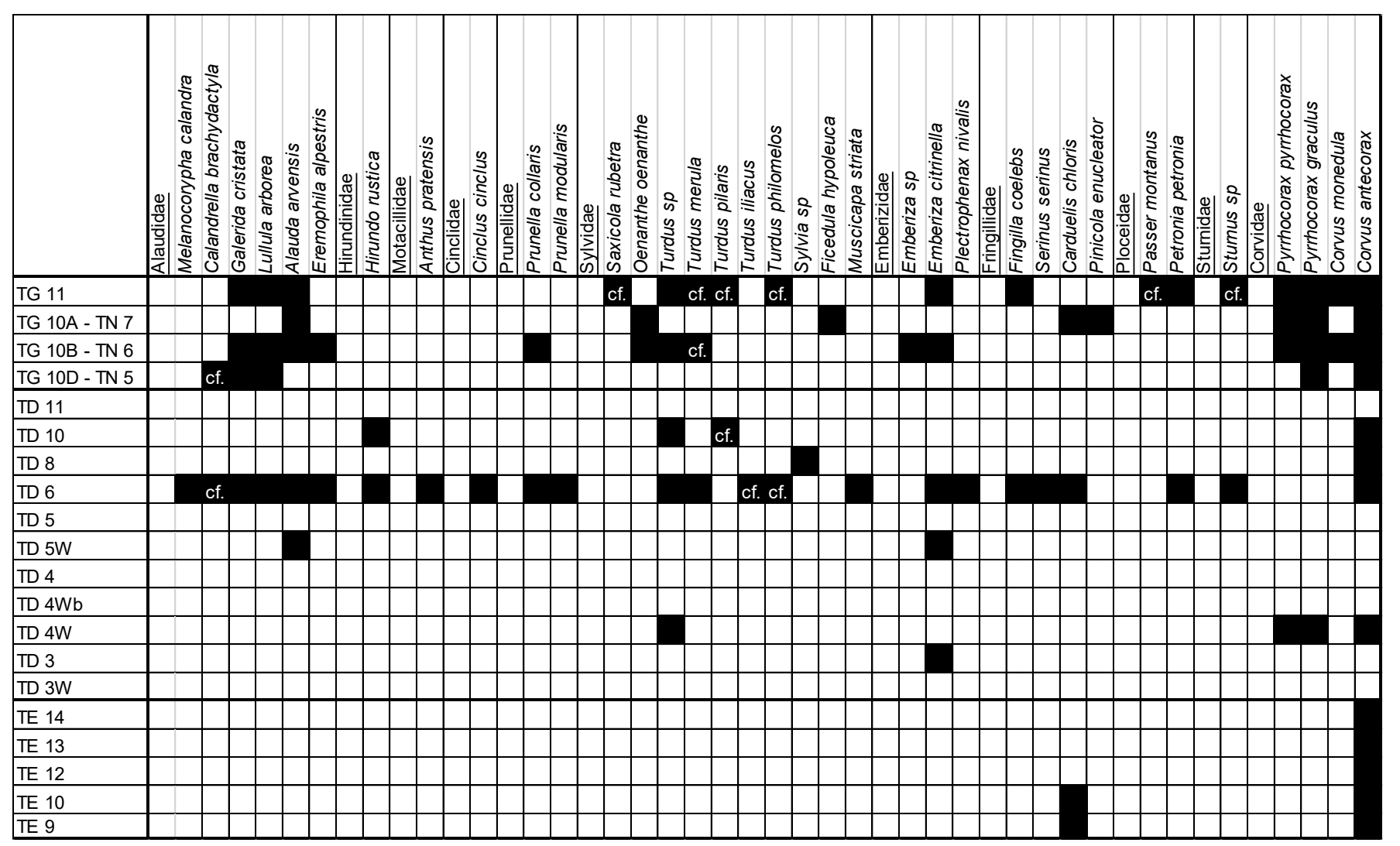

Tabla 2. Distribución por niveles de las aves passeriformes del conjunto de yacimientos de Atapuerca | fuente MADE; AGUIRRE; BASTIR et ál., 2003; SÁNCHEZ MARCO, 2009 
dos y que las aves invernantes no parecen indicar condiciones especialmente frías en el hemisferio norte. También, es evidente que en las proximidades del sistema kárstico había una laguna grande o sistema lagunar o río con aguas remansadas donde se congregaban muchas anátidas, limícolas, numerosas rapaces especializadas en cazar en la superficie del agua, etc.

En la excavación histórica realizada en la cueva de El Castillo se encontraron fósiles de 49 especies de aves (Sánchez Marco, 2006b; 2018). La tabla 3 (pp. 164-165) corresponde a la excavación histórica e incluye niveles desde el aziliense (Holoceno, nivel 4) al musteriense.

En esta localidad han quedado evidencias de especies que no forman parte de la avifauna ibérica actual o son visitantes muy raros. Son: barnacla cariblanca (Branta leucopsis), negrón careto (Melanitta perspicillata), falaropo picogrueso (Phalaropus fulicarius), correlimos oscuro (Calidris maritima), págalo pomarino (Stercorarius pomarinus), charrán ártico (Sterna paradisaea), ampelis europeo (Bombycilla garrulus) y camachuelo picogrueso (Pinicola enucleator). Todas ellas tienen en la actualidad una distribución geográfica más septentrional. Y también se han hallado aquí numerosas especies que no se encuentran en otros yacimientos (SÁNCHEZ MARCO, 2006b; 2018).

La cueva de Santa Catalina se encuentra en la costa vizcaína, y posee niveles magdalenienses y azilienses, del final del Pleistoceno y comienzo de Holoceno. En la tabla 4, el nivel I corresponde al aziliense (Holoceno) y los niveles II y III, al magdaleniense (Elorza, 2014). Con un centenar aproximado de especies, acoge a uno de los conjuntos de especies más ricos de la Península Ibérica (ver tabla 4, pp. 166-168).

Los yacimientos de Santa Catalina y El Castillo han conservado evidencias de muchas aves invernantes que en la actualidad no visitan estas latitudes, en tanto que las aves no migradoras, excepto por la presencia de Lagopus muta, son las mismas que se encontrarían hoy en condiciones naturales. Según el modelo interpretativo planteado (SÁNCHEZ MARCO, 2004), las aves con estatus de residente son las indicadoras de las condiciones climáticas y ambientales en la región del yacimiento paleontológico, y las especies invernantes acusan las condiciones climáticas en las áreas de invernada de latitudes altas. En consecuencia, las aves residentes de estas dos localidades indican ambientes más fríos en el norte peninsular, y las invernantes expresan que el casquete polar norte ha avanzado sobre zonas de invernada, o bien que las condiciones no son aptas para pasar esta fase de su ciclo fenológico anual.

\section{Región mediterránea}

Casablanca 1 es la localidad pleistoceno más antigua de la región mediterránea ibérica. No se sabe mucho de las aves que alberga porque no se

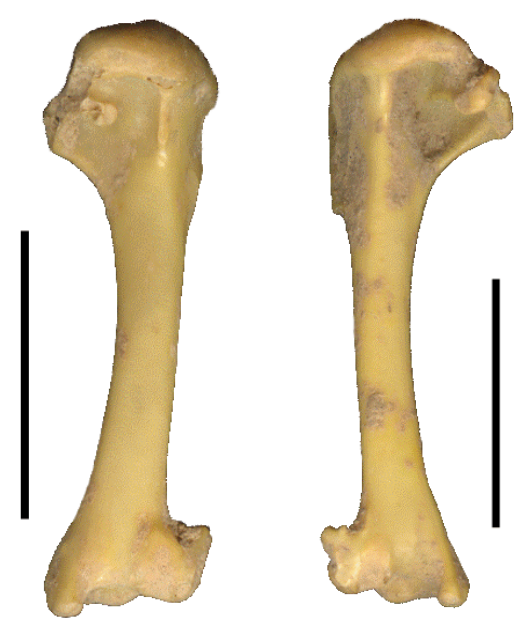

A la izquierda, húmero de Bombycilla garrulus; a la derecha, húmero de Pinicola enucleator. El Castillo. Plesistoceno superior. Escala: $1 \mathrm{~cm}$ | fotos A. Sánchez Marco (2018) 


\begin{tabular}{|c|c|c|c|c|c|c|c|c|c|c|c|c|c|c|}
\hline \multirow{2}{*}{ Taxones } & \multicolumn{14}{|c|}{ Niveles } \\
\hline & 4 & 6 & 7 & 8 & 10 & 12 & 13 & 14 & 16 & 18 & 20 & 22 & Des & Total \\
\hline B. leucopsis & & $2(2)$ & & & & & & $3(1)$ & & & & & & $5(3)$ \\
\hline Tadorna sp. & & & & & & & $1(1)$ & & & & & & & $1(1)$ \\
\hline A. cf. penelope & & & & & & & & & $4(2)$ & & & & & $4(2)$ \\
\hline A.cf. strepera & & & & & & & & & $2(1)$ & & & & & $2(1)$ \\
\hline A. platyrhynchos & & & & $1(1)$ & & & & & $9(3)$ & & & & & $10(4)$ \\
\hline A. cf. acuta & & & & & & & & & $8(3)$ & & & & & $8(3)$ \\
\hline A. querquedula & & & & & & & $2(1)$ & & $4(4)$ & & & & & $6(5)$ \\
\hline A. cf. clypeata & & & & & & & & & $1(1)$ & & & & & $1(1)$ \\
\hline A. fuligula & & & & & & & & & $3(2)$ & & & & & $3(2)$ \\
\hline M. nigra & & & & & & & $1(1)$ & & & & & & & $1(1)$ \\
\hline M. perspicillata & & & & & & & & $1(1)$ & & & & & & $1(1)$ \\
\hline M. fusca & & & & & & & & & $1(1)$ & & & & & $1(1)$ \\
\hline M. serrator & & & & & & & & $1(1)$ & & & & & & $1(1)$ \\
\hline M. merganser & & $1(1)$ & & & & & & & & & & & & $1(1)$ \\
\hline H. albicilla & & & & & & & & & $1(1)$ & & & & & $1(1)$ \\
\hline G. fulvus & & & & & & & & & & & & & $1(1)$ & $1(1)$ \\
\hline A. monachus & & $1(1)$ & & & & & & & & & & & $1(1)$ & $2(2)$ \\
\hline $\begin{array}{l}\text { A. chrysaetos I } \\
\text { adalberti }\end{array}$ & & & & & & & & & & $1(1)$ & & & $2(1)$ & $3(2)$ \\
\hline F. tinnunculus & & & & & & & $2(1)$ & & $11(2)$ & $1(1)$ & $1(1)$ & & & $15(5)$ \\
\hline F. peregrinus & $1(1)$ & & & & & & & & & & & & & $1(1)$ \\
\hline L. mutus & & & & & & & $2(1)$ & & $3(1)$ & $4(1)$ & & & & $9(3)$ \\
\hline A. rufa & & & & & & & & & $1(1)$ & & & & & $1(1)$ \\
\hline P. perdix & & & & & & & $1(1)$ & & $8(1)$ & $1(1)$ & & & & $10(3)$ \\
\hline G. gallus & & $1(1)$ & & & & & & & & & & & & $1(1)$ \\
\hline R. aquaticus & & & & & & & & & $1(1)$ & & & & & $1(1)$ \\
\hline $\begin{array}{l}\text { Charadriiformes } \\
\text { indet. }\end{array}$ & & & & & & & & & $1(1)$ & & & & & $1(1)$ \\
\hline V. vanellus & & & & & & & & & $13(3)$ & & & & & $13(3)$ \\
\hline
\end{tabular}

Tabla 3. Distribución por niveles de las aves de la excavación histórica de la cueva de El Castillo. Continúa en página siguiente | fuente SANCHEZ MARCO, 2006b; 2018

han estudiado todos los restos avianos extraídos del yacimiento. Entre otras especies, aparece una lechuza extinguida, Tyto balearica, la ganga ortega, Pterocles orientalis, que es el único hallazgo en localidades españolas, pero que se halla en otros yacimientos costeros mediterráneos (SÁNCHEZ MARCO, 2004). Cuando se identificaron restos de ibis eremita, Geronticus eremita, en Casablanca 1 (SÁNCHEZ MARCO, 1996), era la primera vez que se publicaba conscientemente un hallazgo de íbises fósiles en el mundo. Esta especie en la actualidad tiene una distribución residual muy distinta de la que tuvo en el pasado. Vuelve a aparecer en el Pleistoceno de dos sitios de la costa ibérica, Quibas (SÁNCHEZ MARCO, 2004) y Gorham (datos inéditos) y en yacimientos de Sicilia (PAVIA, 1999) y Bulgaria (BOEV, 1998; 2002), lo que muestra que estas aves se extendían por el sur de Europa en el Pleistoceno inferior. Quibas y cueva Victoria son dos sitios de Murcia. Quibas está en una zona montañosa del interior, y cueva Victoria, en la costa. Ambos se formaron hace más de un millón de años. En Quibas se encuentra la paleoespecie Palaeocryptonyx donnezani, como en los niveles 


\begin{tabular}{|c|c|c|c|c|c|c|c|c|c|c|c|c|c|c|}
\hline P. apricaria & & & & & & & $1(1)$ & & $5(2)$ & & & & & $6(3)$ \\
\hline P. squatarola & & & & & & & & & $10(2)$ & & & & & $10(2)$ \\
\hline Scolopacidae indet. & & & & & & & & & $1(1)$ & & & & & $1(1)$ \\
\hline Ph. fulicarius & & & & & & & & & $1(1)$ & & & & & $1(1)$ \\
\hline S. rusticola & & & & & & & & & & $1(1)$ & & & & $1(1)$ \\
\hline C. martima & & & & & & & & & $1(1)$ & & & & & $1(1)$ \\
\hline St. pomarinus & & & & & & & & & & $1(1)$ & & & & $1(1)$ \\
\hline L. hyperboreus & & & & & & & & & $1(1)$ & & & & & $1(1)$ \\
\hline St. paradisaea & & & & & & & & & $2(1)$ & & & & & $2(1)$ \\
\hline A. alle & & & & & & & & & $3(2)$ & & & & & $3(2)$ \\
\hline C. livia / oenas & & & & & & & & $2(1)$ & $23(2)$ & & & & & $25(3)$ \\
\hline Strigidae indet. & & $1(1)$ & & & & & & & & & & & & $1(1)$ \\
\hline B. bubo & & & & & & & & & $2(1)$ & & & & & $2(1)$ \\
\hline A. flammeus & & & & & & & $1(1)$ & & $2(2)$ & & & & & $3(3)$ \\
\hline Passeriformes indet. & & & & & & & & & $8(3)$ & & & & & $8(3)$ \\
\hline B. garrullus & & & & & & & & & $1(1)$ & & & & & $1(1)$ \\
\hline T. cf. pilaris & & & & & & & & & $5(2)$ & & & & & $5(2)$ \\
\hline T. cf. iliacus & & & & & & & & & $1(1)$ & & & & & $1(1)$ \\
\hline T. cf. philomelos & & & & & & & & & $1(1)$ & & & & & $1(1)$ \\
\hline P. enucleator & & & & & & & & & $7(3)$ & & $3(2)$ & & & $10(5)$ \\
\hline P. petronia & & & & & & & & & $2(1)$ & & & & & $2(1)$ \\
\hline P. pica & & & & & & & & & $3(2)$ & $1(1)$ & & & & $4(3)$ \\
\hline P. pyrrhocorax & & & & $3(2)$ & & $2(1)$ & $1(1)$ & $3(1)$ & $9(3)$ & $2(1)$ & $4(1)$ & & & 24 \\
\hline P. graculus & & $\begin{array}{l}13 \\
(3)\end{array}$ & $\begin{array}{l}3 \\
(2)\end{array}$ & $5(2)$ & $\begin{array}{l}1 \\
(1)\end{array}$ & & $2(1)$ & $8(2)$ & $62(9)$ & $5(2)$ & & $1(1)$ & & $\begin{array}{l}100 \\
(23)\end{array}$ \\
\hline C. monedula & & & & & & & & & $2(1)$ & & & & & $2(1)$ \\
\hline C. corone / frugilegus & & & & & & & & $2(2)$ & & & & & & $2(2)$ \\
\hline C. corax & & & & & $\begin{array}{l}1 \\
(1)\end{array}$ & & $2(1)$ & $2(1)$ & $3(2)$ & $3(1)$ & & & & $11(6)$ \\
\hline Aves indet. & & & & & & & $1(1)$ & & $3(2)$ & & & & & $4(3)$ \\
\hline Total & $1(1)$ & $\begin{array}{l}19 \\
(9)\end{array}$ & $\begin{array}{l}3 \\
(2)\end{array}$ & $9(5)$ & $\begin{array}{l}2 \\
(2)\end{array}$ & $2(1)$ & $\begin{array}{l}17 \\
(12)\end{array}$ & $22(10)$ & $\begin{array}{l}229 \\
(75)\end{array}$ & $\begin{array}{l}20 \\
(11)\end{array}$ & $8(4)$ & $1(1)$ & $4(3)$ & $\begin{array}{l}337 \\
(136)\end{array}$ \\
\hline
\end{tabular}

Tabla 3. Distribución por niveles de las aves de la excavación histórica de la cueva de El Castillo Viene de página anterior | fuente SÁNCHEZ

inferiores de Elefante. Quibas y Victoria poseen ornitocenosis de tipo mediteMARCO, 2006b; 2018 rráneo, con presencia de Alectoris, entre otros taxones (SÁNCHEZ MARCO, $2004 ; 2009 ; 2015)$. En ambos yacimientos han fosilizado aves de ambientes forestales. Este carácter del paisaje es especialmente acusado en el caso de cueva Victoria, situada muy cerca del mar. Aquí aparece gallo lira, Tetrao tetrix, junto con dos especies de picapinos, Picus viridis y Dryocopus martius (SÁNCHEZ MARCO, 2004; 2015).

En la depresión de Guadix-Baza se encuentra el yacimiento del Pleistoceno inferior de Huéscar 1. Tiene la singularidad de que aparece el guión de codornices, Crex crex. El conjunto ornítico está compuesto mayoritariamente por aves acuáticas, casi todas invernantes -como zampullines (Tachybaptus ruficollis), diversas especies de ánades (Anas spp.), cercetas (Anas querquedula), patos colorados (Netta rufina) y varios porrones (Aythya spp.), negrón común (Melanitta nigra), serreta mediana (Mergus serrator)-, excepto unas pocas residentes, como Bubo bubo y Alectoris (SÁNCHEZ MARCO, 2004; 
Tabla 4. Distribución por niveles de las aves de Santa Catalina. Continúa en páginas siguientes | fuente ELORZA, 2014

\begin{tabular}{|c|c|c|c|c|c|c|c|c|}
\hline & \multicolumn{2}{|c|}{ Nivel I } & \multicolumn{2}{|c|}{ Nivel II } & \multicolumn{2}{|c|}{ Nivel III } & \multicolumn{2}{|l|}{ Total } \\
\hline & NISP & MNI & NISP & MNI & NISP & NMI & NISP & MNI \\
\hline Cygnus cygnus (Cisne cantor) & & & 2 & 1 & & & 2 & 1 \\
\hline Cygnus sp. & & & 1 & & & & 1 & \\
\hline Cygnus sp. / Anser sp. & & & 1 & & & & 1 & \\
\hline $\begin{array}{l}\text { Anser cf. albifrons (Ansar careto } \\
\text { grande) }\end{array}$ & & & 1 & 1 & & & 1 & 1 \\
\hline Anser anser (Ansar común) & 1 & 1 & 1 & 1 & & & 2 & 2 \\
\hline Anser anser / fabalis & 2 & 1 & 5 & 1 & & & 7 & 2 \\
\hline Anser sp. & 20 & & 52 & & 2 & & 74 & \\
\hline Anser sp. / Branta sp. & 34 & & 126 & & 25 & & 185 & \\
\hline Anser albifrons / Branta leucopsis & & & 1 & & & & 1 & \\
\hline $\begin{array}{l}\text { Branta leucopsis (Barnacla cari- } \\
\text { blanca) }\end{array}$ & 3 & 1 & 2 & 1 & 1 & 1 & 6 & 3 \\
\hline Branta sp. & 4 & & 3 & & & & 7 & \\
\hline Tadorna tadorna / ferruginea (Tarro) & 1 & 1 & 2 & 1 & & & 3 & 2 \\
\hline Anas penelope / strepera & & & 1 & 1 & & & 1 & 1 \\
\hline Anas crecca (Cerceta común) & 2 & 1 & 8 & 3 & 1 & 1 & 11 & 5 \\
\hline $\begin{array}{l}\text { Anas querquedula (Cerceta carre- } \\
\text { tona) }\end{array}$ & & & 4 & 3 & & & 4 & 3 \\
\hline Anas crecca / querquedula & 7 & & 19 & & 1 & & 27 & \\
\hline Anas platyrhynchos (Anade real) & 28 & 5 & $92(1)$ & 12 & 10 & 4 & 130 & 21 \\
\hline Anas platyrhynchos / strepera & 1 & 1 & 2 & 1 & & & 3 & 2 \\
\hline Anas acuta (Anade rabudo) & 1 & 1 & & & & & 1 & 1 \\
\hline Anas acuta / platyrhynchos & 10 & 2 & 22 & 5 & 2 & 1 & 34 & 8 \\
\hline Anas clypeata (Cuchara común) & & & 2 & 1 & & & 2 & 1 \\
\hline Anas clypeata / penelope & & & 3 & 1 & & & 3 & 1 \\
\hline Anas sp. & 47 & & 174 & & 10 & & 231 & \\
\hline Aythya fuligula (Porrón europeo) & 2 & 1 & 3 & 1 & 1 & 1 & 6 & 3 \\
\hline Aythya ferina (Porrón moñudo) & & & 2 & 1 & & & 2 & 1 \\
\hline Aythya ferina / fuligula & & & 1 & 1 & & & 1 & 1 \\
\hline Aythya ferina / marila & & & 2 & 1 & & & 2 & 1 \\
\hline Aythya sp. & 4 & & 7 & & 1 & & 12 & \\
\hline Somateria mollissima (Eider común) & & & & & $3(1)$ & 1 & 3 & 1 \\
\hline Somateria cf. spectabilis (Eider real) & & & & & 9 & 1 & 9 & 1 \\
\hline Somateria sp. & & & & & 1 & & 1 & \\
\hline Polysticta stelleri (Eider de Steller) & & & 4 & 1 & 7 & 3 & 11 & 4 \\
\hline Clangula hyemalis (Havelda) & & & 1 & 1 & 9 & 2 & 10 & 3 \\
\hline Melanitta nigra (Negrón común) & 3 & 1 & 10 & 2 & 14 & 5 & 27 & 8 \\
\hline $\begin{array}{l}\text { Melanitta fusca (Negrón especula- } \\
\text { do) }\end{array}$ & & & 3 & 1 & 2 & 1 & 5 & 2 \\
\hline Melanitta sp. & 1 & & 8 & & 2 & & 11 & \\
\hline
\end{tabular}




\begin{tabular}{|c|c|c|c|c|c|c|c|c|}
\hline & \multicolumn{2}{|c|}{ Nivel I } & \multicolumn{2}{|c|}{ Nivel II } & \multicolumn{2}{|c|}{ Nivel III } & \multicolumn{2}{|l|}{ Total } \\
\hline & NISP & MNI & NISP & MNI & NISP & NMI & NISP & MNI \\
\hline $\begin{array}{l}\text { Bucephala clangula (Porrón oscu- } \\
\text { lado) }\end{array}$ & & & 1 & 1 & 1 & 1 & 2 & 2 \\
\hline Mergus serrator (Serreta mediana) & 1 & 1 & & & $2(1)$ & 1 & 3 & 2 \\
\hline Mergus sp. & & & 1 & & & & 1 & 1 \\
\hline Lagopus lagopus (Lagópodo común) & & & 1 & 1 & 10 & 4 & 11 & 5 \\
\hline Lagopus sp. & & & 8 & & 47 & & 49 & \\
\hline Alectoris sp. (Perdiz) & 1 & & & & & & 1 & \\
\hline Perdix perdix (Perdiz pardilla) & 1 & 1 & 2 & 1 & 3 & 1 & 6 & 3 \\
\hline Gavia arctica (Colimbo ártico) & & & & & 2 & 1 & 2 & 1 \\
\hline $\begin{array}{l}\text { Podiceps auritus (Zampullin } \\
\text { cuellirojo) }\end{array}$ & 1 & 1 & 2 & 1 & & & 4 & 2 \\
\hline Fulmarus glacialis (Fulmar boreal) & & & 2 & 1 & 1 & 1 & 3 & 2 \\
\hline Puffinus gravis (Pardela capirotada) & 3 & 1 & & & & & 3 & 1 \\
\hline $\begin{array}{l}\text { Puffinus puffinus (Pardela picho- } \\
\text { neta) }\end{array}$ & 1 & 1 & & & & & 1 & 1 \\
\hline Puffinus cf. olsoni (Pardela de lava) & & & 1 & 1 & 1 & 1 & 2 & 2 \\
\hline Puffinus holeae (Pardela dunar) & 2 & 1 & 2 & 1 & 1 & 1 & 5 & 3 \\
\hline Puffinus sp. & & & 2 & & & & 2 & \\
\hline Morus bassanus (Alcatraz atlántico) & 1 & 1 & 16 & 2 & 5 & 2 & 22 & 5 \\
\hline $\begin{array}{l}\text { Phalacrocorax carbo (Cormorán } \\
\text { grande) }\end{array}$ & 2 & 1 & 1 & 1 & 2 & 1 & 5 & 3 \\
\hline $\begin{array}{l}\text { Phalacrocorax aristoteles (Cormorán } \\
\text { moñudo) }\end{array}$ & 1 & 1 & 1 & 1 & & & 2 & 2 \\
\hline Ardea cinerea (Garza real) & & & $1(1)$ & 1 & & & 1 & 1 \\
\hline $\begin{array}{l}\text { Haliaetus cf. albicilla (Pigargo } \\
\text { europeo) }\end{array}$ & 1 & 1 & & & 2 & 1 & 3 & 2 \\
\hline $\begin{array}{l}\text { Gypaetus barbatus (Quebrantahue- } \\
\text { sos) }\end{array}$ & & & 1 & 1 & 5 & 1 & 6 & 2 \\
\hline Accipiter gentilis (Azor común) & & & 1 & 1 & & & 1 & 1 \\
\hline $\begin{array}{l}\text { Falco cf. tinnunculus (Cernícalo } \\
\text { vulgar) }\end{array}$ & 1 & 1 & 2 & 1 & & & 3 & 2 \\
\hline Falco sp. & & & 3 & & 3 & & 6 & \\
\hline Fulica atra / cristata (Focha) & 1 & 1 & 3 & 1 & & & 4 & 2 \\
\hline Grus grus (Grulla común) & & & 1 & 1 & & & 1 & 1 \\
\hline Tetrax tetrax (Sisón común) & 3 & 1 & 16 & 3 & & & 19 & 4 \\
\hline Otis tarda (Avutarda común) & 3 & 1 & 6 & 1 & & & 9 & 2 \\
\hline $\begin{array}{l}\text { Haematopus ostralegus (Ostreo } \\
\text { euroasiático) }\end{array}$ & & & & & 1 & 1 & 1 & 1 \\
\hline Charadrius apricaria / squatarola & & & 1 & 1 & 1 & 1 & 2 & 2 \\
\hline Vanellus vanellus (Avefría europea) & 3 & 1 & 2 & 1 & & & 5 & 2 \\
\hline $\begin{array}{l}\text { Calidris cf. alpina (Correlimos } \\
\text { común) }\end{array}$ & & & & & $4(1)$ & 1 & 4 & 1 \\
\hline
\end{tabular}

Tabla 4. Distribución por niveles de las aves de Santa Catalina. Viene de página anterior | fuente ELORZA, 2014 
. Distribución por niveles de las aves de Santa Catalina. Viene de página 167 | fuente ELORZA, 2014

\begin{tabular}{|c|c|c|c|c|c|c|c|c|}
\hline & \multicolumn{2}{|c|}{ Nivel I } & \multicolumn{2}{|c|}{ Nivel II } & \multicolumn{2}{|c|}{ Nivel III } & \multicolumn{2}{|l|}{ Total } \\
\hline & NISP & MNI & NISP & MNI & NISP & NMI & NISP & MNI \\
\hline Scolopax rusticola (Chocha perdiz) & 5 & 1 & 2 & 1 & 1 & 1 & 8 & 3 \\
\hline Limosa limosa / lapponica & 1 & 1 & & & & & 1 & 1 \\
\hline $\begin{array}{l}\text { cf. Numenius phaeopus (Zarapito } \\
\text { trinador) }\end{array}$ & & & 1 & 1 & 1 & 1 & 2 & 2 \\
\hline cf. Numenius arquata (Zarapito real) & & & 1 & 1 & & & 1 & 1 \\
\hline $\begin{array}{l}\text { Tringa cf. erythropus (Archibebe } \\
\text { oscuro) }\end{array}$ & 1 & 1 & & & & & 1 & 1 \\
\hline Tringa totanus (Archibebe común) & & & 2 & 1 & & & 2 & 1 \\
\hline Larus canus / tridactylus (Gaviota) & 1 & 1 & 2 & 1 & & & 3 & 2 \\
\hline Larus argentatus-cachinnans-fuscus & & & 1 & 1 & 17 & 3 & 18 & 4 \\
\hline Larus marinus / hyperboreus & 1 & 1 & 1 & 1 & 27 & 3 & 29 & 5 \\
\hline Sterna hirundo (Charrán común) & 1 & 1 & 4 & 1 & & & 5 & 2 \\
\hline Uria aalge / lomvia (Arao) & 3 & 2 & 27 & 3 & 9 & 2 & 39 & 7 \\
\hline Alca torda (Alca común) & 2 & 1 & 5 & 1 & 6 & 1 & 13 & 3 \\
\hline Alca torda / Uria sp. & & & 9 & & 4 & & 13 & \\
\hline Pinguinus impennis (Alca grande) & 2 & 1 & $7(1)$ & 2 & $87(5)$ & 8 & 96 & 11 \\
\hline Alle alle (Mérgulo atlántico) & & & 2 & 1 & 1 & 1 & 3 & 2 \\
\hline $\begin{array}{l}\text { Fratercula arctica (Frailecillo atlán- } \\
\text { tico) }\end{array}$ & 4 & 1 & & & & & 4 & 1 \\
\hline Columba livia / oenas & 1 & 1 & 4 & 2 & 3 & 1 & 8 & 4 \\
\hline Columba palumbus (Paloma torcaz) & & & 1 & 1 & & & 1 & 1 \\
\hline Bubo scandiacus (Búho nival) & & & 3 & 1 & 49 & 5 & 52 & 6 \\
\hline Bubo bubo / scandiacus & 1 & & 8 & & 89 & & 98 & \\
\hline Strix aluco (Cárabo común) & 1 & 1 & & & & & 1 & 1 \\
\hline Apus apus / pallidus & & & 1 & 1 & & & 1 & 1 \\
\hline Delichon urbica / Riparia riparia & & & 1 & 1 & & & 1 & 1 \\
\hline Turdus iliacus (Zorzal alirrojo) & 1 & 1 & & & & & 1 & 1 \\
\hline Turdus iliacus / philomelos & 2 & & 2 & & & & 4 & 3 \\
\hline Turdus sp. & 16 & & 14 & & & & 25 & \\
\hline Pica pica (Urraca) & & & 1 & 1 & & & 1 & 1 \\
\hline $\begin{array}{l}\text { Pyrrhocorax graculus (Chova } \\
\text { piquigualda) }\end{array}$ & & & 3 & 1 & $12(1)$ & 3 & 15 & 4 \\
\hline $\begin{array}{l}\text { Pyrrhocorax pyrrhocorax (Chova } \\
\text { piquirroja) }\end{array}$ & & & 1 & 1 & 3 & 1 & 4 & 2 \\
\hline Pyrrhocorax sp. & & & 6 & & 11 & & 17 & \\
\hline Pyrrhocorax sp. I Corvus monedula & & & 4 & & 5 & & 9 & \\
\hline Corvus monedula (Grajilla común) & & & 1 & & & & 1 & \\
\hline $\begin{array}{l}\text { Corvus corone / frugilegus (Corneja } \\
\text { / Graja) }\end{array}$ & 1 & 1 & & & & & 1 & 1 \\
\hline Corvus corax (Cuervo común) & 1 & 1 & 3 & 1 & 9 & 2 & 13 & 4 \\
\hline Fringilla sp. & 1 & 1 & & & & & 1 & 1 \\
\hline
\end{tabular}


2009). Tal comunidad de aves implica que el depósito se formó cerca de una masa de agua extensa y profunda, con vegetación en los bordes. La presencia del negrón común puede significar que los inviernos eran muy crudos en latitudes altas de Europa.

El yacimiento de la cueva de Nerja es muy rico en especies, muchas se han hallado en niveles holocenos (BOESSNECK; DRIESCH, 1980; EASTHAM, 1986; HERNÁNDEZ, 1995; CORTÉS; MORALES; SIMÓN et ál., 2008).

En el peñón de Gibraltar, por encima de donde transcurre una de las mayores rutas migratorias de aves del mundo, se encuentra el yacimiento hasta el presente más rico en este grupo de vertebrados, Gorham's cave. Se realizó un primer estudio de una muestra amplia (EASTHAM, 1968), pero no tan grande como se obtendría tiempo después, y posteriormente una tesis doctoral (Cooper, 1999). En una siguiente fase, se han estudiado casi todos los restos óseos que se han ido extrayendo en las sucesivas campañas de excavación. Se han publicado varios artículos que se apoyan en alguna medida en las aves de las últimas excavaciones (Finlayson; Giles; Rodríguez et ál., 2006; Finlayson; Carrión; Brown et ál., 2011; Finlayson; Brown; Blasco et ál., 2012; Finlayson; Finlayson; Giles-Guzmán et ál., 2016; Blasco; Finlayson; Rosell et ál., 2014; Blasco; Rosell; Rufà et ál., 2016), si bien hasta ahora no se había dado a conocer la lista completa. En la tabla 5 (pp. 170-171), se presenta la lista de taxones estudiados e identificados hasta la fecha por el autor del presente artículo. El nivel IV corresponde al Paleolítico medio, y el nivel III, al Paleolítico superior.

Las aves fosilizadas en Gorham componen una muestra excepcionalmente variada y rica de las ornitocenosis mediterráneas ibéricas. Son muy abundantes las rapaces diurnas, las aves marinas, las acuáticas invernantes y las aves propias de la región mediterránea. Como sucede en otros yacimientos, en Gorham también hay especies procedentes del norte de Europa, como Bubo scandiacus, que irrumpen en zonas meridionales, probablemente como respuesta al deterioro climático en latitudes altas. En la costa occidental de Iberia, en la península de Peniche, la gruta da Furninha ha proporcionado un conjunto amplio de aves, entre las que sobresalen las acuáticas invernantes, el alga grande y un elevado número de rapaces (FIGUEIREDO; CUNHA; SOUSA et ál., 2017).

\section{Especies fósiles}

En varios yacimientos peninsulares del Pleistoceno se han identificado y descrito algunas especies de aves distintas de las actuales. Pinguinus impennis aparece en varios yacimientos costeros ibéricos al final del Pleistoceno y principio del Holoceno; Corvus antecorax y Perdix palaeoperdix se han citado en localidades del Pleistoceno inferior y medio (SÁNCHEZ MARCO, 2004); Aegypius prepyrenaicus fue descrito por Hernández (2001) mediante

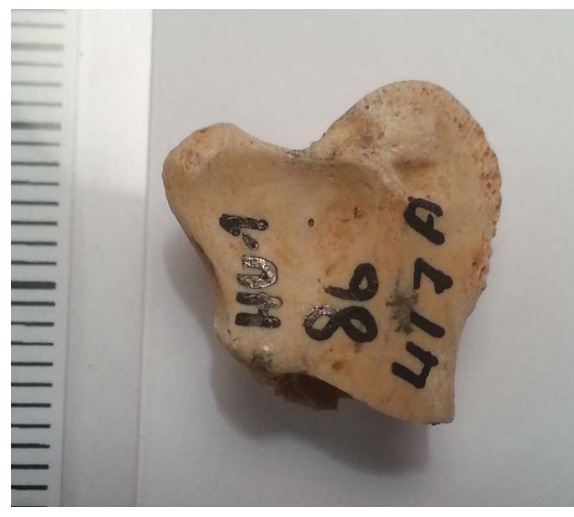

Parte proximal de carpometacarpo de Bubo bubo. Huéscar 1. Pleistoceno inferior | foto A. Sánchez Marco 


\begin{tabular}{|c|c|c|c|}
\hline Especies halladas & $\begin{array}{l}\text { Solo en el } \\
\text { nivel III }\end{array}$ & $\begin{array}{l}\text { Solo en el } \\
\text { nivel IV }\end{array}$ & $\begin{array}{l}\text { En ambos } \\
\text { niveles }\end{array}$ \\
\hline $\begin{array}{l}\text { Gavia stellata } \\
\text { Puffinus cf. puffinus } \\
\text { Puffinus sp. } \\
\text { Calonectris diomedea } \\
\text { Sula bassana } \\
\text { Phalacrocorax aristotelis } \\
\text { Phalacrocorax carbo } \\
\text { Plegadis falcinellus } \\
\text { Geronticus eremita } \\
\text { Anatidae indet. } \\
\text { Tadorna sp. } \\
\text { Anas sp. } \\
\text { Marmaronetta angustirostris } \\
\text { Netta rufina } \\
\text { Aythya ferina } \\
\text { Clangula hyemalis } \\
\text { Melanitta nigra } \\
\text { Mergus serrator } \\
\text { Milvus milvus } \\
\text { Milvus migrans } \\
\text { Accipiter gentilis } \\
\text { Accipiter nisus } \\
\text { Buteo buteo } \\
\text { Buteo lagopus } \\
\text { Hieraaetus fasciatus } \\
\text { Aquila cf. chrysaetos } \\
\text { Haliaeetus albicilla } \\
\text { Gyps melitensis } \\
\text { Gypaetus barbatus } \\
\text { Circus cyaneus } \\
\text { Falco naumanni } \\
\text { Falco tinnunculus } \\
\text { Falco subbuteo } \\
\text { Falco peregrinus } \\
\text { Alectoris rufa } \\
\text { Coturnix coturnix } \\
\text { Tetrax tetrax } \\
\text { Haematopus ostralegus } \\
\text { Himantopus himantopus } \\
\text { Pluvialis squatarola } \\
\text { Charadrius hiaticula } \\
\text { Numenius phaeopus } \\
\text { Limosa limosa } \\
\text { Tringa totanus } \\
\text { Calidris alpina } \\
\text { Calidris maritima } \\
\text { Larus cf. argentatus } \\
\text { Larus marinus / hyperboreus } \\
\text { Stercorarius parasiticus } \\
\text { Catharactes skua } \\
\text { Pinguinus impennis } \\
\text { Fratercula arctica } \\
\text { Columba livia s. oenas } \\
\text { Asio flammeus } \\
\text { Otus scops }\end{array}$ & $\begin{array}{l}- \\
- \\
- \\
- \\
- \\
- \\
x \\
- \\
x \\
x \\
x \\
- \\
x \\
x \\
x \\
x \\
- \\
- \\
- \\
- \\
x \\
x \\
- \\
- \\
x \\
- \\
- \\
- \\
- \\
- \\
- \\
- \\
- \\
- \\
- \\
- \\
- \\
x \\
- \\
x \\
x \\
- \\
- \\
x \\
x \\
- \\
- \\
- \\
- \\
x \\
- \\
x \\
- \\
- \\
- \\
\end{array}$ & 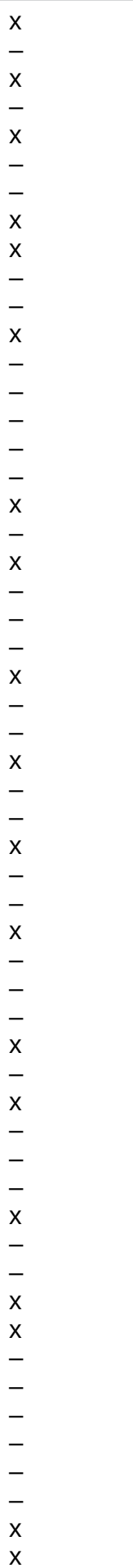 & 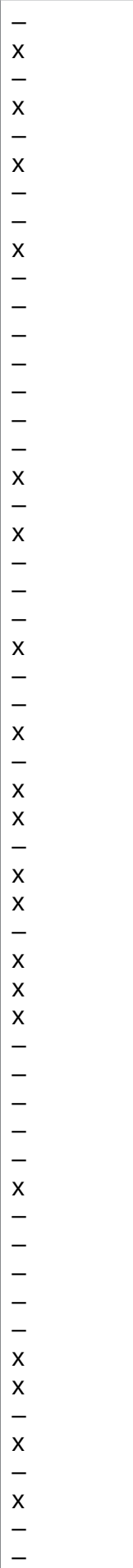 \\
\hline
\end{tabular}
ves de Gorham's cave (campaña de excavación 1999-2005). Datos inéditos.Continúa en página siguiente 


\begin{tabular}{|l|l|l|l|}
\hline Especies halladas & Solo en el & Solo en el & En ambos \\
& nivel III & nivel IV & niveles \\
\hline Bubo scandiacus & $\mathrm{x}$ & - & - \\
Athene noctua & $\mathrm{x}$ & - & - \\
Tyto alba & - & $\mathrm{x}$ & - \\
Apus apus & - & - & $\mathrm{x}$ \\
Apus melba & - & $\mathrm{x}$ & - \\
Galerida cristata & - & - & $\mathrm{x}$ \\
Lullula arborea & - & - & $\mathrm{x}$ \\
Alauda arvensis & $\mathrm{x}$ & - & - \\
Hirundo rustica & - & - & $\mathrm{x}$ \\
Ptyonoprogne rupestris & - & - & $\mathrm{x}$ \\
Motacilla alba & - & $\mathrm{x}$ & - \\
Motacilla flava & - & $\mathrm{x}$ & - \\
Anthus spinoletta & - & - & $\mathrm{x}$ \\
Anthus campestris & - & - & $\mathrm{x}$ \\
Anthus pratensis & - & - & $\mathrm{x}$ \\
Prunella collaris & - & $\mathrm{x}$ & - \\
Erithacus rubecula & - & $\mathrm{x}$ & - \\
Phoenicurus ochruros & - & - & $\mathrm{x}$ \\
Oenanthe oenanthe & $\mathrm{x}$ & - & - \\
Oenanthe hispanica & - & $\mathrm{x}$ & - \\
Turdus spp. & - & - & $\mathrm{x}$ \\
Ficedula hypoleuca & - & $\mathrm{x}$ & - \\
Parus major & - & $\mathrm{x}$ & - \\
Certhia sp. & - & $\mathrm{x}$ & - \\
Emberiza hortulana & $\mathrm{x}$ & - & - \\
Emberiza citrinella & - & $\mathrm{x}$ & - \\
Emberiza calandra & - & $\mathrm{x}$ & - \\
Fringilla coelebs & - & - & $\mathrm{x}$ \\
Carduelis chloris & - & $\mathrm{x}$ & - \\
Carduelis flammea & - & $\mathrm{x}$ & - \\
Pyrrhula pyrrhula & - & $\mathrm{x}$ & - \\
Passer sp. & $\mathrm{x}$ & - & - \\
Sturnus sp. & - & $\mathrm{x}$ & - \\
Pyrrhocorax pyrrhocorax & - & - & $\mathrm{x}$ \\
Pyrrhocorax graculus & - & - & $\mathrm{x}$ \\
Corvus monedula & - & $\mathrm{x}$ & - \\
Corvus corone & - & - & - \\
Corvus corax & - & - & $\mathrm{x}$ \\
& & & \\
\hline & & & \\
\hline
\end{tabular}

un fragmento proximal de ulna procedente de Gabasa I (Pleistoceno supe-

Tabla 5. Distribución en los niveles IV y III de las aves de Gorham's cave (campaña de excavación 1999-2005). Datos inéditos. Viene de página rior); un tarsometatarso que carece del extremo distal, procedente de Cal anterior

Guardiola (Pleistoceno inferior) fue descrito como Bubo ibericus por Meijer, Pavia, Madurell-Malapiera et ál. (2016); Tyto balearica se cita en Casablanca 1 (Pleistoceno inferior) (SÁNCHEZ MARCO, 1999; 2004); Gyps melitensis se menciona como cronoespecie en tres yacimientos (SÁNCHEZ MARCO, 2007); Palaeocryptonyx donnezani se identifica en dos yacimientos del Pleistoceno inferior, y en uno de ellos se describe Bonasa nini (SÁNCHEZ MARCO, 2009). Esta última es una galliforme forestal. Las aves de hábitats boscosos son las peor representadas en el registro fósil. Su hallazgo es muy interesante. Los últimos ejemplares vivos del alca gigante o grande, Pinguinus 
impennis, moraban en Islandia y Groenlandia, razón por la que algunos autores la consideraron especie indicadora de climas fríos, incluso cuando la encontraron en las costas mediterráneas. Sin embargo, las localidades pleistocenas donde aparece -Devil's Tower (BATE, 1928), Gorham (EASTHAM, 1968), Ibex (COOPER, 2000), Nerja (CORTÉS; MORALES; SIMÓN et ál., 2008), Figueira Brava (MOURER-CHAUVIRÉ; ANTUNES, 1991)- presentan en todos los casos contextos orníticos de carácter mediterráneo. Además, su presencia en el Mediterráneo no se debe a individuos divagantes, porque los frecuentes restos de individuos juveniles indican que esta especie criaba también en el sur de Europa.

En cuanto a Corvus antecorax y Perdix palaeoperdix, éstas se describen en el Pleistoceno de Francia y posteriormente se identifican en varios yacimientos franceses y españoles (SÁNCHEZ MARCO, 2004). Se describen como formas de talla inferior a la de los representantes actuales respectivos, Corvus corax y Perdix perdix. Pero con el aumento de hallazgos de restos de estos animales, la separación entre tallas ha ido desapareciendo, y se debe considerar a tales formas como sinónimas de las actuales, o como cronoespecies (formas fósiles con algún carácter diagnóstico particular y que encajan en una secuencia temporal con una especie actual, sin valor taxonómico) (SÁNCHEZ MARCO, 2007; 2009).

En ocasiones, puede tener interés mantener la denominación de la paleoespecie para conservar la información del carácter diferencial (como una talla diferente) que a lo largo del tiempo se pierde. Un inconveniente es que siempre hay un lapso de tiempo en el que converge el carácter diferencial de la cronoespecie con la forma actual, y el límite entre ambas es imposible de establecer. La ulna sobre la que se erige Aegypius prepyrenaicus pertenece a un Gyps de talla superior a la actual de Gyps fulvus, por lo que este taxón fue transferido a Gyps melitensis, junto con restos previamente identificados como Gyps fulvus o Gyps sp. provenientes de La Cuevona y Gorham (SÁNCHEZ MARCO, 2007). Gyps melitensis se describió mediante restos procedentes del Pleistoceno de Malta como una especie de tamaño considerablemente superior al del buitre leonado actual, Gyps fulvus. Posteriormente, se identificó en numerosos yacimientos europeos, tanto continentales como en islas del Mediterráneo. Sin embargo, tras revisar el material original, se observó que la talla de los fósiles tipo no era tan grande como se había publicado y se considera como cronoespecie de Gyps fulvus (SÁNCHEZ MARCO, 2007).

El caso de Bubo ibericus es distinto. El hueso en que se basa esta especie solo se ha comparado con Bubo, en lugar de hacerlo con todas las Strigiformes. Al observar la figura y las medidas en el artículo de Meijer, Pavia, Madurell-Malapiera et ál. (2016), se comprende que no pertenece a Bubo. Probablemente es de un cárabo (Strix). Paleocryptonyx donnezani se halla en localidades europeas del Plioceno y Pleistoceno inferior. Su presencia en 
Quibas y en Elefante (Atapuerca) se entiende como una pervivencia de este animal en el Pleistoceno inferior de la Península Ibérica. La identificación de Tyto balearica requiere matizaciones -véase la discusión en Sánchez Marco (en prensa)- porque está ligada, en parte, a los distintos conceptos de especie que utilizan los diversos autores.

Una especie paleontológica (también denominada paleoespecie o especie fósil) es una especie de la que solo se tienen evidencias fósiles. Este término se aplica a los restos de unos individuos muertos que poseían en común características exclusivas y novedosas (autoapomorfías). Una especie fósil no es necesariamente una especie biológica extinguida. Una especie biológica comprende a todos los individuos que potencialmente se puedan cruzar y tener descendencia fértil (MAYR, 1942). Cuando se trata con restos fósiles, es imposible saber si los caracteres distintivos que se toman como apomórficos describen a la totalidad de una comunidad reproductiva de individuos-especie biológica-. Es decir, no es posible saber si varias especies fósiles morfológicamente parecidas, cuyos individuos vivían al mismo tiempo, constituían o no especies biológicas distintas. Sobre todo, es imposible saber si los caracteres distintivos de una especie fósil implican una comunidad reproductiva cerrada respecto a especies fósiles ancestrales o a especies biológicas (actuales) filogenéticamente próximas.

Tyto balearica se describe con material de varias localidades baleares del Pleistoceno inferior, morfológicamente igual a la actual Tyto alba, pero de talla más grande. En trabajos posteriores, se la identifica en yacimientos europeos continentales, del Mioceno superior (MN12), Plioceno y Pleistoceno inferior (MOURER-CHAUVIRÉ; SÁNCHEZ MARCO, 1988; SÁNCHEZ MARCO, 2001; en prensa). El hallazgo en Córcega y Cerdeña de huesos de una forma de Tyto de mayor tamaño que la Tyto alba actual ha conducido a Louchart (2002) y a Pavia y Mourer-Chauviré (2011) a considerar que Tyto balearica se debería asignar solo a los fósiles de las Baleares y a los de Córcega y Cerdeña. Hay dos puntos a considerar aquí. Uno es que las proporciones y tallas de las lechuzas de Córcega y Cerdeña no se corresponden con las medidas de los fósiles tipo de Baleares. El otro es que estos autores toman a Tyto balearica como una especie biológica y, además, se dejan llevar por la idea de que se trataba de una especie que se distribuía por las islas mediterráneas -como si para una lechuza fuera posible desplazarse directamente entre las Baleares y Córcega y Cerdeña- (véase la discusión en Sánchez Marco, en prensa). En mi opinión, en el Pleistoceno existió una Tyto en Córcega y Cerdeña con algunos huesos de mayor talla que la lechuza común actual. También hubo una forma de mayor talla en las Baleares, que recibió el nombre de Tyto balearica, y que es diferente de la Córcega y Cerdeña. También hay unas lechuzas en el continente europeo, desde el Mioceno superior, que coinciden en talla con Tyto balearica. Si los fósiles continentales y los de las Baleares pertenecieron o no a diferentes especies biológicas, no se puede abordar con los medios de 
la paleontología. La sistemática y taxonomía se basan en criterios anatómicos (recientemente también moleculares). Hay que excluir criterios extrínsecos, tales como la cronología o la geografía, del proceso de descripción de un taxón. Este tipo de criterios a veces están presentes implícita o explícitamente porque se confunde la secuencia de las fases del procedimiento de construcción de la sistemática zoológica. La taxonomía es una fase independiente y previa a las explicaciones e hipótesis sobre las condiciones del proceso de diversificación taxonómica.

\section{Faunas insulares}

Las faunas insulares son generalmente muy pobres en especies, pero también es bien conocido que las islas oceánicas muy alejadas de las masas continentales presentan una alta proporción de endemismos. Tanto en las Baleares como en las Canarias, se han descrito algunas especies nuevas, pero no se observa una tasa de especiación comparable a la que se da en otras islas, quizá como consecuencia de su proximidad a los continentes (SÁNCHEZ MARCO, 2004). Una alta tasa de endemicidad en aves aparentemente requiere distancias mayores entre las islas y los continentes que las necesarias para otros grupos de vertebrados.

\section{Islas Baleares y Pitiusas}

En los yacimientos del Pleitoceno de la cova de Canet, Pedrera de S'Onix (ambos localizados en Mallorca) y Barranc de Binigaus (en Menorca) se encontraron los huesos de una lechuza relacionada con la lechuza común actual, Tyto alba, pero de mayor tamaño, que se la describió como Tyto balearica (MOURER-CHAUVIRÉ; ALCOVER; MOYÀ et ál., 1980). Una lechuza que se supuso que constituía un endemismo insular, pero que después fue hallada en yacimientos continentales de España y Francia (MOURER-CHAUVIRÉ; SÁNCHEZ MARCO, 1988), como se ha mencionado más arriba. En la cova de Ca Na Reia (Ibiza) se describió Puffinus nestori y se identificaron Palaeocryptonyx y otras especies actuales (ALCOVER, 1989). El yacimiento más rico en número de huesos y en especies es la localidad ibicenca de Es Pouàs. Se han identificado más de 70 especies o géneros (FLORIT; MOURER-CHAUVIRÉ; ALCOVER, 1989; ALCOVER; MCMINN, 1992; GUERRA; MCMINN; ALCOVER, 2013; GUERRA, 2015). Los estudios de los restos avianos no han concluido, pues la cantidad de éstos es considerable. McMinn, Palmer y Alcover (2005) describieron Rallus eivisiensis.

\section{Islas Canarias}

El registro fósil de las islas Canarias es muy rico en restos óseos, pero es muy pobre en especies. La naturaleza volcánica de los terrenos de este archipiélago limita las condiciones favorables para la sedimentación y conservación de los huesos de los cadáveres. La lista de aves registradas en 
yacimientos canarios es más corta que en las Baleares y Pitiusas, lo que también es así en lo que respecta a las aves actuales. Una causa probable es la relativa juventud de las Canarias, que limita el tiempo para posibles colonizaciones. Alguna influencia ha de tener el hecho de que a escasas decenas de kilómetros, sobre la costa occidental africana, discurre una de las mayores rutas migratorias de aves, lo que cabe esperar que sea fuente de colonizadores ocasionales.

La mayor parte de los yacimientos se forman en tubos volcánicos y en depósitos eólicos. En tales formaciones eólicas se encuentran sobre todo esqueletos de aves marinas. En algunas localidades hay enormes cantidades de restos, pero casi siempre pertenecientes a muy pocas especies. Grandes extensiones de este tipo, muy ricas en fósiles, son relativamente frecuentes en Fuerteventura, como en el barranco del Pecenescal (SÁNCHEZ MARCO, 2003; 2010), aunque hay otros puntos en otros lugares, como Timbaiba, en Lanzarote, con incontables restos óseos pertenecientes a dos pardelas, Puffinus holeae y Calonectris diomedea (BISBAL-CHINESTA; ISERN ATARES; MARTÍN REGUEIRAS et ál., 2015). Se han descrito varias especies extinguidas: Carduelis triasi (ALCOVER; FLORIT, 1987); Puffinus olsoni (MCMINN; JAUME; ALCOVER, 1990); Puffinus holeae (WALKER; WRAGG; HARRISON, 1990); Coturnix gomerae (JAUME; MCMINN; ALCOVER, 1993); y Emberiza alcoveri (RANDO; LÓPEZ; SEGUÍ, 1999). Las passerinas pueden ser paleoendemismos insulares. Puffinus holeae, no, y Puffinus olsoni, quizá tampoco porque la primera se encuentra en el Pleistoceno de Figueira Brava (MOURER-CHAUVIRÉ; ANTUNES, 2000) y de Santa Catalina (ELORZA, 2014), y la segunda se identifica con dudas también en (ELORZA, 2014). Más recientemente, se ha descrito Carduelis aurelioi (RANDO; ALCOVER; ILLERA, 2010) hallado en Tenerife, con una datación en el límite Pleistoceno/ Holoceno.

Las cáscaras de huevo de gran tamaño que aparecen en tres yacimientos del norte de Lanzarote han suscitado polémica en cuanto al grupo aviano que las hubiera producido (ROTHE, 1964; SAUER; ROTHE, 1972; GARCÍATALAVERA, 1990; BÁEZ, 1982; 1992). Un elemento importante en el debate fue el hallazgo de un fragmento de hueso que García-Talavera (1990) atribuye a un pelagornítido. El autor del artículo presente, tras revisar directamente el mencionado fragmento óseo, que se conserva en el Museo de la Naturaleza y el Hombre (Santa Cruz de Tenerife), de tres centímetros y medio de longitud, considera que no pertenece a un ave. Estos yacimientos se pensaba que se habían formado durante el Mioceno superior o el límite Mioceno/ Plioceno (ROTHE, 1964; SAUER; ROTHE, 1972; GARCÍA-TALAVERA, 1990), pero dataciones recientes los sitúan en el Plioceno inferior (LOMOSCHITZ; SÁNCHEZ MARCO; HUERTAS et ál., 2016). Últimamente, se han retomado los estudios de los materiales procedentes de estas localidades. Además del estudio geológico de Lomoschitz, Sánchez Marco, Huertas et ál. (2016),

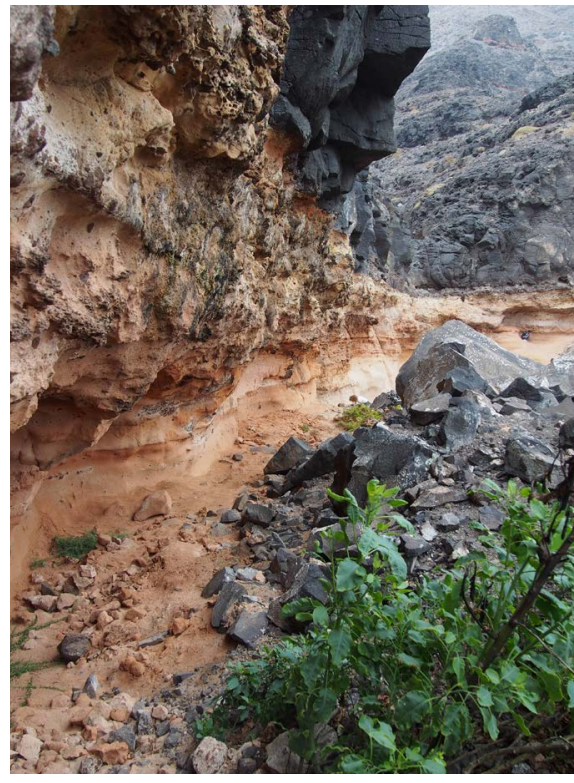

Vista del yacimiento de Valle Chico, Lanzarote. Plioceno inferior | foto A. Sánchez Marco 
se ha publicado un análisis de isótopos estables (LAZZERINI; LÉCUYER; AMIOT et ál., 2016).

Un compendio de los trabajos realizados sobre materiales avianos de las islas Canarias, bastante completo hasta la fecha de su publicación, así como varios aspectos de las condiciones y dinámicas que en el pasado han configurado la composición de las ornitocenosis actuales se puede ver en Sánchez Marco (2010). Las islas Canarias están cerca del continente africano, por cuya costa, además, transcurre una ruta migratoria de gran importancia. Esto quizá es la causa principal que establece notables diferencias con respecto a las comunidades orníticas de islas del Pacífico y del Índico, y a las consecuencias de la llegada de los primeros humanos a tales islas (OLSON; JAMES, 1982; MILLENER, 1999; STEADMAN, 1989; 2006; STEADMAN; ROLETT, 1996). Los escasos nuevos datos no parecen contradecir las líneas generales que se expusieron: (1) baja proporción de endemicidad en la ornitofauna actual; (2) baja proporción de especies endémicas extinguidas; (3) quizá con una excepción, las formas endémicas no pierden la capacidad de vuelo ni aumentan su talla; y (4) una tasa muy baja de desaparición de especies, tanto antes de la llegada de humanos, como después. En este sentido, no hay evidencias concluyentes de que las actividades cinegéticas o agrícolas de los primeros grupos humanos que se establecen en Canarias ni, posteriormente, de los europeos, hayan causado la desaparición de alguna especie de ave, contrariamente a lo que sucede en las islas del Î́ndico y del Pacífico.

Este trabajo se ha realizado con la ayuda de la Agencia Estatal de Investigación (AEI) de España y los Fondos de Desarrollo Regional Europeo de la Unión Europea (CGL2016-76431-P). 


\section{BIBLIOGRAFÍA}

- AlCOVER, J. A. (1989) Les aus fòssils de la Cova de Ca Na Reia. Endins [en línea], vol. 14/15, 1989, pp. 95-100 <http://ibdigital.uib.es/greenstone/collect/ endins/index/assoc/Endins 1/989v14_1/5p095.dir/ Endins_1989v14_15p095.pd $\bar{f}>$ [Consultā: 12/04/2018]

- ALCOVER, J. A.; FLORIT, F. (1987) Una nueva especie de Carduelis (Fringillidae) de La Palma. Vieraea, vol. 17, 1987, pp. 75-86

- ALCOVER, J. A.; MCMINN, M. (1992) Presència de l'àguila marina Haliaeetus albicilla (Linnaeus 1758) al jaciment espeleologic quaternari d'es Pouàs (Sant Antoni de Portmany, Eivissa). Endins, vol. 17-18, 1992, pp. 81-87

- BISBAL-CHINESTA, J. F.; ISERN ATARES, A.; MARTÍN REGUEIRAS, A.; BETANCORT, J. F. (2015) Aves marinas (Procellariidae) del Pleistoceno del yacimiento paleontológico de la Montaña de Timbaiba (Lanzarote, Islas Canarias). En XIII EJIP (Encuentro de Jóvenes Investigadores en Paleontología). Conference Proceedings/Libro de resúmenes, Cercedilla (Madrid), 15-18 de abril de 2015, pp. 60-66

- BLASCO, R.; FINLAYSON, C.; ROSELL, J.; SÁNCHEZ MARCO, A.; FINLAYSON, S.; FINLAYSON, G.; NEGRO, J. J.; GILES PACHECO, F.; RODRÍGUEZ VIDAL, J. (2014) The earliest pigeon fanciers. Scientific Reports, vol. 4, 2014, article number: 5971, 2014

- BLASCO, R.; ROSELL, J.; RUFÀ, A.; SÁNCHEZ MARCO, A.; FINLAYSON, C. (2016) Pigeons and choughs, a usual resource for the Neanderthals in Gibraltar. Quaternary International, vol. 421, 2016, pp. 62-77

- BOESSNECK, J.; DRIESCH, A. (1980) Tierknochenfunde aus vier südspanischen Höhlen. Studien über frühe Tierknochenfunde von der Iberischen Halbinsel, vol. 7, 1980, pp. 1-83

- BOEV, Z. (1998) Presence of Bald Ibis (Geronticus Wagler, 1832) (Threskiornithidae-Aves) in the late Pliocene of Bulgaria. Geologica Balcanica, vol. 28, n. ${ }^{\circ} 1-2,1988$, pp. 45-52

- BOEV, Z. (2002) Additional material of Geronticus balcanicus Boev, 1998, and Precision of the Age of the type locality. Acta Zoologica Bulgarica, vol. 52, n. ${ }^{\circ}$ 2, 2002, pp. 53-58

- BOEV, Z.; SPASSOV, N. (2009) First record of ostriches (Aves, Struthioniformes, Struthionidae) from the late Miocene of Bulgaria with taxonomic and zoogeographic discussion. Geodiversitas, vol. 31, n. ${ }^{\circ} 3$, 2009, pp. 493-507

- BONADONNA, F. P.; VILLA, I. M. (1984) Estudio geocronológico del volcanismo de Las Higueruelas. En El medio físico de Castilla-La Mancha, vol. III. Albacete: Junta de Comunidades de Castilla-La Mancha, 1984, pp. 249-253
- BRAVO, A. M.; YÉBENES, M.; MARTíN, H. (2009) Cáscaras de huevo de tipo Aepyornis del Mioceno superior de Alicante (España). Aproximación filogenética. Revista Española de Paleontología, vol. 24, n. ${ }^{\circ}$ 1, 2009, pp. 47-58

- BRODKORB, P. (1964) Catalogue of fossil birds. Part 2 (Anseriformes through Galliformes). Bulletin of the Florida State Museum, Biological Sciences, vol. 8, 1964, pp. 195335

- BRUIJN, H. DE; DAAMS, R.; DAXNER-HÖCK, G.; FAHLSBUSCH, V.; GINSBURG, L.; MEIN, P.; MORALES, J. (1992) Report of the RCMNS working group on fossil mammals, Reisensburg 1990. Newsletters of Stratigraphy, vol. 26, n. ${ }^{\circ} 2 / 3,1992$, pp. $65-118$

- BUFFETAUT, E. (2008) First evidence of the giant bird Gastornis from southern Europe: a tibiotarsus from the Lower Eocene of Saint-Papoul (Aude, southern France). Oryctos, vol. 7, 2008, pp. 75-82

- BUFFETAUT, E.; PEREDA-SUBERBIOLA, X.; CORRAL, C.; ANGST, D. (2015) First Iberian record of the giant Late Cretaceous Gargantuavis. Libro de resúmenes I Congreso Internacional Las aves en la historia natual, en la prehistoria y en la historia (Origen, evolución y domesticación), 23 al 27 de septiembre de 2015, Lisboa, p. 12

- CENIZO, M. (2012) Review of putative Phorusrhacidae from the Cretaceous and Paleogene of Antarctica: new records of ratites and palagornithid birds. Polish Polar Research, vol. 33, n. ${ }^{\circ} 3,2012$, pp. 225-244

- CHENEVAL, J.; ADROVER, R. (1993) L'avifaune du Miocène supérieur d'Aljezar B (Los Aljezares, province de Teruel, Espagne). Systématique et paléoécologie. Paleontologia i Evolució, vol. 26-27, 1993, pp. 133-144

- COOPER, J. H. (1999) Late Pleistocene avifaunas of Gibraltar and their palaeoenvironmental significance. University of London. Tesis doctoral inédita

- COOPER, J. H. (2000) A preliminary report on the Pleistocene avifauna of Ibex cave, Gibraltar. En FINLAYSON, J. C.; FINLAYSON, G.; FA, D. (ed.) Gibraltar during the Quaternary. Gibraltar: Gibraltar Government Heritage Publications, 2000

- CORTÉS, M.; MORALES, A.; SIMÓN, M. D.; BERGADÀ, M. M.; DELGADO, A.; LÓPEZ, P.; LÓPEZ, J. A.; LOZANO, M. C.; RIQUELME, J. A.; ROSELLÓ, E.; SÁNCHEZ MARCO, A.; VERA, J. L. (2008) Palaeoenvironmental and cultural dynamics of the coast of Málaga (Andalusia, Spain) during the Upper Pleistocene and Early Holocene. Quaternary Science Reviews, vol. 27, n. ${ }^{\circ}$ 23/24, 2008, pp. 2176-2193

- EASTHAM, A. (1968) The avifauna of Gorham's Cave, Gibraltar. Bulletin of the London Institute of Archaeology, vol. 7, 1968, pp. 37-42 
- EASTHAM, A. (1986) The birds of the Cueva de Nerja. En JORDÁ, J. F. (ed.) La prehistoria de la Cueva de Nerja (Málaga). Nerja: Patronato de la Cueva de Nerja, 1986, pp. 109-131

- ElORZA, M. (2014) Explotación de aves marinas en el Tardiglaciar del golfo de Bizkaia. Las aves de Santa Catalina. Kobie, vol. 4, 2014, pp. 263-296

- FEDUCCIA, A. (1974) Another Old World vulture from the New World. Wilson Bulletin, vol. 86, 1974, pp. 251255

- FINLAYSON, C.; GILES, F.; RODRÍGUEZ, J.; FA, D.; GUTIÉRREZ, J. M.; SANTIAGO, A.; FINLAYSON, G.; ALLUÉ, E.; BAENA, J.; CÁCERES, I.; CARRIÓN, J. S.; FERNÁNDEZ, Y.; GLEED-OWEN, C.; JIMÉNEZ, F. J.; LÓPEZ, P.; LÓPEZ, J. A.; RIQUELME, J. A.; SÁNCHEZ MARCO, A.; GILES, F.; BROWN, K.; FUENTES, N.; VALARINO, C. A.; VILLALPANDO, A.; STRINGER, C. B.; MARTÍNEZ, F.; SAKAMOTO, T. (2006) Late survival of Neandertals at the southernmost extreme of Europe. Nature, vol. 443, 2006, pp. 850-853

- FINLAYSON, C.; CARRIÓN, J.; BROWN, K.; FINLAYSON, G.; SÁNCHEZ-MARCO, A.; FA, D.; RODRÍGUEZ-VIDAL, J.; FERNÁNDEZ, S.; FIERRO, E.; BERNAL-GÓMEZ, M.; GILES-PACHECO, F. (2011) The Homo habitat niche: using the avian fossil record to depict ecological characteristics of Palaeolithic Eurasian hominins. Quaternary Science Reviews, vol. 30, 2011, pp. 1525-1532

- FINLAYSON, C.; BROWN, K.; BLASCO, R.; ROSELL, J.; NEGRO, J. J.; BORTOLOTTI, G. R.; FINLAYSON, G.; SÁNCHEZ MARCO, A.; GILES PACHECO, F.; RODRÍGUEZ VIDAL, J.; CARRIÓN, J. S.; FA, D. A.; RODRÍGUEZ LLANES, J. M. (2012) Birds of a Feather: Neanderthal Exploitation of Raptors and Corvids. PLOS ONE [en línea], vol. 7(9):e45927, 2012 <https://www.ncbi.nlm.nih.gov/pmcl articles/PMC3444460/> [Consulta: 01/04/2018]

- FINLAYSON, C.; FINLAYSON, S.; GILES-GUZMÁN, F.; SÁNCHEZ MARCO, A.; FINLAYSON, G.; JENNINGS R.; GILES-PACHECO, F.; RODRÍGUEZ VIDAL, J. (2016) Using birds as indicators of Neanderthal environmental quality: Gibraltar and Zafarraya compared. Quaternary International [en línea], vol. 421, 9, noviembre 2016, pp. 32-45 <https://www.sciencedirect.com/science/article/pii/ S1040618215011568> [Consulta: 01/04/2018]

- FLORIT, F.; MOURER-CHAUVIRÉ, C.; ALCOVER, J. A. (1989) Els ocells pleistocènics d'es Pouàs, Eivissa. Nota preliminar. Butlletí de la Institució Catalana d'Història Natural, vol. 56, 1989, pp. 35-46

- GAILLARD, C. (1908) Les oiseaux des Phosphorites de Quercy. Annales de l'Université de Lyon, vol. 23, 1908, pp. $1-178$
- GUERRA, C. (2015) Avifauna del Pleistoceno superiorHoloceno de la Pitiusas: passeriformes y sus depredadores. Tesis doctoral inédita. Universidad de Salamanca

- GUERRA, C.; MCMINN, M.; ALCOVER, J. A. (2013) The Upper Pleistocene-Holocene raptorial bird guild from Eivissa Island (Pityusic Archipelago, Western Mediterranean Sea). Geobios, vol. 46, 2013, pp. 491-502

- HADDRATH, O.; BAKER, J. (2001) Complete mitochondrial DNA genome sequence of extinct birds: ratite phylogenetics and the vicariance biogeography hypothesis. Proccedings of the Royal Society of London B, vol. 268, 2001, pp. 939-945

- HARRISON, C. J. O. (1983) A new wader, Recurvirostridae (Charadriiformes), from the early Eocene of Portugal. Ciências da Terra (UNL), vol. 7, 1983, pp. 9-16

- HARSHMAN, J.; BRAUN, E. L.; BRAUN, M. J.; HUDDLESTON, C. J.; BOWIE, R. C. K.; CHOJNOWSKI, J. L.; HACKETT, S. J.; HAN, K. L.; KIMBALL, R. T.; MARKS, B. D.; MIGLIA, K. J; MOORE, W. S.; REDDY, S.; SHELDON, F. H.; STEADMAN, D. W.; STEPPAN, S. J.; WITT, C. C.; YURI, T. (2008) Phylogenomic evidence for multiple losses of flight in ratite birds. Proceedings of the National Academy of Sciences, vol. 105, n. ${ }^{\circ} 36,2008$, pp. 13462-13467

- HERNÁNDEZ, F. (1995) Cueva de Nerja (Málaga): las aves de las campañas de 1980 y 1982. Trabajos sobre la cueva de Nerja, vol. 5, 1995, pp. 221-293

- HERNÁNDEZ, F. (2001) A new species of vulture (Aves, Aegypiinae) from the upper Pleistocene of Spain. Ardeola, vol. 48, n. ${ }^{\circ} 1,2001$, pp. 47-53

- HOU, L. (1984) The Aragonian vertebrate fauna of Xiacaowan, Jiangsu 2. Aegypinae (Falconiformes, Aves). Vertbr PalAsiat, vol. 22, n. ${ }^{\circ} 1,1984$, pp. 14-20

- HOU, L.; ZHOU, Z.; ZHANG, F.; LI, J. (2000) A new vulture from the Miocene of Shandong, eastern China. Vertbr PalAsiat, vol. 38, n. ${ }^{\circ}$ 2, 2000, pp. 104-110

- HOWARD, H. (1966) Two fossil birds from the Lower Miocene of South Dakota. Los Angeles County Museum of Natural History Contributions to Science, vol. 107, 1966, pp. 1-8

- JAUME, D.; MCMINN, M.; ALCOVER, J. A. (1993) Fossil birds from the Bujero del Silo, La Gomera (Canary Islands), with a description of a new species of quail (Galliformes: Phasianidae). Boletim do Museu Municipal do Funchal, supl. 2, 1993, pp. 147-165

- LACASA RUIZ, A. (1986) Nota preliminar sobre el hallazgo de restos óseos de un ave fósil en el yacimiento neocomiense del Montsec, prov. de Lérida, España. Ilerda, vol. 47,1986 , pp. 203-206 
- LACASA RUIZ, A. (1989) Nuevo género de ave fósil del yacimiento neocomiense del Montsec (provincia de Lérida, España). Estudios geológicos, vol. 45, 1989, pp. 417-425

- LAZZERINI, N.; LÉCUYER, C.; AMIOT, R.; ANGST, D.; BUFFETAUT, E.; FOUREL, F.; DAUX, V.; FLANDROIS, J. P.; BETANCORT, J. F.; SÁNCHEZ MARCO, A.; LOMOSCHITZ, A. (2016) Oxygen isotope fractionation between bird eggshell calcite and body water: application to fossil eggs from Lanzarote (Canary Islands). Science of Nature, vol. 103 (9/10), art. 81, 2016

- LI, Z.; CLARKE, J. A.; ZHOU, Z.; DENG, T. (2016) A new Old World vulture from the late Miocene of China sheds light on Neogene shifts in the past diversity and distribution of the Gypaetinae. The Auk, vol. 133, 2016, pp. 615-625

- LOMOSCHITZ, A.; SÁNCHEZ MARCO, A.; HUERTAS, M. J.; BETANCORT, J. F.; ISERN, A.; SANZ, E.; MECO, J. (2016) A reappraisal of the stratigraphy and chronology of Early Pliocene palaeontological sites from Lanzarote island containing fossil terrestrial animals. Journal of African Earth Sciences, vol. 123, 2016, pp. 338-349

- LOUChART, A. (2002) Les oiseaux du Pléistocène de Corse et de quelques localités sardes. Écologie, évolution, biogéographie et extinctions. Documents des Laboratoires de Géologie de Lyon, vol. 155, 2002, pp. 1-287

- MADE, J. V. D.; AGUIRRE, E.; BASTIR, M.; FERNÁNDEZ JALVO, Y.; HUGUET, R.; LAPLANA, C.; MÁRQUEZ, B.; MARTÍNEZ, C.; MARTINÓN, M.; ROSAS, A.; RODRÍGUEZ, J.; SÁNCHEZ MARCO, A.; SARMIENTO, S.; BERMÚDEZ DE CASTRO, J. M. (2003) El registro paleontológico y arqueológico de los yacimientos de la Trinchera del Ferrocarril en la Sierra de Atapuerca. En Homenaje a Remmert Daams. Coloquios de Paleontología, vol. ext. I, 2003, pp. 345-372

- MAYR, E. (1942) Systematics and the origin of species. New York: Columbia University Press, 1942, pp. 334

- MCMINN, M.; JAUME, D.; ALCOVER, J. A. (1990) Puffinus olsoni n. sp.: nova espècie de baldritja recentment extingida provinent de depòsits espeleològics de Fuerteventura i Lanzarote (Illes Canàries, Atlàntic Oriental). Endins, vol. 16, 1990, pp. 63-71

- MCMINN, M.; PALMER, M.; ALCOVER, J. A. (2005) A new species of rail (Aves: Rallidae) from the Upper Pleistocene and Holocene of Ibiza (Pityusic Islands, western Mediterranean). Ibis, vol. 147, n. ${ }^{\circ} 4$, 2005, pp. 706-716

- MEIJER, H. J. M.; PAVIA, M.; MADURELL-MALAPIERA, J.; ALBA, D. M. (2016) A revision of fossil eagle owls (Aves, Strigiformes, Bubo) from Europe and the description of a new species, Bubo ibericus, from Cal Guardiola (NE Iberian Peninsula). Historical Biology, 2016, pp. 822-832 <https:// doi.org/10.1080/08912963.2016.1247836> [Consulta: 1/4/ 2018]

- MEIN, P.; DAUPHIN, Y. (1995) Des coquilles d'oeufs de type Aepyornis dans le Bassin de Teruel (Pliocène basal, Espagne). Neues Jahrbuch für Geologie und Paläontologie Monatshafte, vol. 3, 1995, pp. 182-191

- MILLENER, P. R. (1999) The history of the Chatham Islands' bird fauna of the last 7000 years-a chronicle of change and extinction. Smithsonian Contributions to Paleobiology, vol. 89, 1999, pp. 85-109

- MLÍKOVSKÝ, J. (2002) Cenozoic birds of the world. Part 1: Europe. Prague: Ninox Press, 2002

- MONTOYA, P. (1997) Los hiénidos (Carnívora, Mammalia) del Mioceno superior (Turoliense inferior) de Crevillente 2 (provincia de Alicante, España). Revista Española de Paleontología, vol. 12, n. ${ }^{\circ} 2,1997$, pp. 265273

- MORALES, A.; POZO, M.; SILVA, P. G.; DOMINGO, M. S.; LÓPEZ, R.; ÁLVAREZ, M. A.; ANTÓN, M.; MARTÍN, C.; QUIRALTE, V.; SALESA, M. J.; SÁNCHEZ, I. M.; AZANZA, B.; CALVO, J. P.; CARRASCO, P.; GARCÍA-PAREDES, I.; KNOLL, F.; HERNÁNDEZ, M.; HOEK, L. V. D.; MERINO, L.; MEULEN, J. A. V. D.; MONTOYA, P.; PEIGNÉ, S.; PELÁEZ, P.; SÁNCHEZ MARCO, A.; TURNER, A.; ABELLA, J.; ALCALDE, G. M.; ANDRÉS, M.; MIGUEL, D. D.; CANTALAPIEDRA, J. L.; FRAILE, S.; GARCÍA, B. A.; GÓMEZ, A. R.; LÓPEZ, P.; OLIVER, A.; SILÍCEO, G. (2008) El sistema de yacimientos de mamíferos miocenos del Cerro de los Batallones, cuenca de Madrid: estado actual y perspectivas. En ESTEVE, J.; MELÉNDEZ, G. (ed.) Palaeontologica Nova. Zaragoza: Departamento de Ciencias de la Tierra, Área y Museo de Paleontología, Universidad de Zaragoza, 2008, pp. 41-117 (Publicaciones del Seminario de Paleontología de Zaragoza, vol. 8)

- MOURER-CHAUVIRÉ, C. (1992) The Galliformes (Aves) from the Phosphorites du Quercy (France): systematics and biostratigraphy. En CAMPBELL, K. E. (ed.) Papers in Avian Paleontology. Los Ángeles: Natural History Museum of Los Angeles County, 1992, pp. 67-95

- MOURER-CHAUVIRÉ, C.; ALCOVER, J. A.; MOYÀ, S.; PONS, J. (1980) Une nouvelle forme insulaire d'effraie géante, Tyto balearica n. sp. (Aves, Strigiformes), du PlioPléistocène des Baléares. Geobios, vol. 13, 1980, pp. 803811

- MOURER-CHAUVIRÉ, C.; ANTUNES, M. T. (2000) L'avifaune pléistocène et holocène de Gruta da Figueira Brava (Arrábida, Portugal). En ANTUNES, M. T. (ed.) Colloquium Last neanderthals in Portugal. Memórias da Academia das Ciências de Lisboa, vol. 38, 2000, pp. 129159 
- MOURER-CHAUVIRÉ, C.; SÁNCHEZ MARCO, A. (1988) Présence de Tyto balearica (Aves, Strigiformes) dans des gisements continentaux du Pliocène de France et d'Espagne. Geobios, vol. 21, n. ${ }^{\circ}$ 5, 1988, pp. 639-644

- OLSON, S. L.; JAMES, H. F. (1982) Fossil birds from the Hawaiian Islands: evidence for wholesale extinction by man before western contact. Science, 217, 1982, pp. 633-635

- PATNAIK, R.; SAHNI, A.; CAMERON, D.; PILLANS, B.; CHATRATH, P.; SIMONS, E.; WILLIAMS, M.; BIBI, F. (2009) Ostrich-like eggshells from a 10.1 million-yr-old Miocene ape locality, Haritalyangar, Himachal Pradesh, India. Current Science, vol. 96, n. ${ }^{\circ}$ 11, 2009, pp. 1485-1495

- PAVIA, M. (1999) The Middle Pleistocene avifauna of Spinagallo cave (Sicily, Italy): preliminary report. Smithsonian Contributions to Paleobiology, vol. 89, 1999, pp. $125-127$

- PAVIA, M.; MOURER-CHAUVIRÉ, C. (2011) Redescription of Tyto sanctialbani Lydekker, 1893 (Aves, Strigiformes), from its type locality of La Grive-Saint-Alban (middle Miocene, France). Journal of Vertebrate Paleontology, vol. 31, 2011, pp. 1093-1101

- RANDO, J. C.; LÓPEZ, M.; SEGUÍ, B. (1999) A new species of extinct flightless passerine (Emberizidae: Emberiza) from the Canary Islands. The Condor, vol. 101, 1999, pp. 1-13

- RANDO, J. C.;ALCOVER, J. A.; ILLERA, J. C. (2010) Disen tangling ancient interactions: a new exticnt passerine provi des insights on character displacement among extinct and extant island finches. PloS ONE, vol. 5, n. ${ }^{\circ}$ 9, 2010, pp. e12956

- RAYMO, M. E. (1991) Global climate change: a three million year perspective. NATO ASI Series, vol. I, n. ${ }^{\circ} 3$, 1991, pp. 207-223

- RAYMO, M. E.; RUDDIMAN, W. F.; CLEMENT, B. M. (1986) Pliocene-Pleistocene paleoceanography of the North Atlantic at Deep Sea Drilling Project site 609. Initial Report Deep Sea Drilling Project, vol. 94, 1986, pp. 895901

- SÁNCHEZ MARCO, A. (1990) A new bustard (Otididae; Aves) from the early Pliocene of Layna (Soria, Spain). Paleontologia i Evolució, vol. 23, 1990, pp. 223-229

- SÁNCHEZ MARCO, A. (1995a) Tertiary avian localities of Portugal. En MLÍKOVSKÝ, J. (ed.) Tertiary Avian Localities of Europe. Acta Universitatis Carolinae Geologica, 39 (3/4), Univerzita Karlova, Praga, 1995, pp. 699-701

- SÁNCHEZ MARCO, A. (1995b) Tertiary avian localities of Spain. En MLÍKOVSKÝ, J. (ed.) Tertiary Avian Localities of Europe. Acta Universitatis Carolinae Geologica, 39 (3/4), Univerzita Karlova, Praga, 1995, pp. 719-732
- SÁNCHEZ MARCO,A. (1999a) Catálogo paleornitológico del Terciario ibérico y balear. Estudios geológicos, vol. 55 , n. ${ }^{\circ} 3-4,1999$, pp. 163-171

- SÁNCHEZ MARCO, A. (1999b) Implications of the avian fauna for paleoecology in the Early Pleistocene of the Iberian Peninsula. Journal of Human Evolution, vol. 37, n. ${ }^{\circ}$ 3/4, 1999, pp. 375-388

- SÁNCHEZ MARCO, A. (1999c) Aves del yacimiento mesopleistoceno de Galería (sierra de Atapuerca). Patrones ecológicos en el Pleistoceno medio: 211-224. En CARBONELL, E.; ROSAS, A.; DíEZ, J. C. (ed.) Atapuerca: ocupaciones humanas y paleoecología del yacimiento de Galería. Junta de Castilla y León, Consejería de Educación y Cultura, 1999 (Arqueología en Castilla y León: memorias; vol. 7)

- SÁNCHEZ MARCO, A. (2001) Strigiformes from the Neogene of Spain. Ibis, vol. 143, 2001, pp. 313-316

- SÁNCHEZ MARCO, A. (2003) Nuevo hallazgo de aves marinas del Pleistoceno de Fuerteventura (Islas Canarias). Coloquios de Paleontología, vol. ext. I, 2003, pp. 627-636

- SÁNCHEZ MARCO, A. (2005) Aves del Plioceno superior de la meseta sur ibérica: una asociación ornítica aparentemente cuaternaria. Revista Española de Paleontología, vol. 20, n. ${ }^{\circ}$ 2, 2005, pp. 143-157

- SÁNCHEZ MARCO, A. (2006a) Miophasianus and Palaeoperdix (Galliformes, Aves) from three Miocene localities of Spain. Estudios geológicos, vol. 62, n. ${ }^{\circ} 1,2006$, pp. $249-256$

- SÁNCHEZ MARCO, A. (2006b) Aves fósiles del Auriñaciense (Pleistoceno superior) de El Castillo (Cantabria, España). En MAÍLLO, J. M.; BAQUEDANO, E. (ed.) Miscelanea en homenaje a Victoria Cabrera. Zona Arqueológica, 7, vol. I, 2006, pp. 114-121

- SÁNCHEZ MARCO, A. (2007) New occurrences of the extinct vulture Gyps melitensis (Falconiformes, Aves) and a reappraisal of the paleospecies. Journal of Vertebrate Paleontology, vol. 27, n. ${ }^{\circ} 4,2007$, pp. 1057-1061

- SÁNCHEZ MARCO, A. (2009) New Iberian galliforms and reappraisal of some Pliocene and Pleistocene Eurasian taxa. Journal of Vertebrate Paleontology, vol. 29, n. $^{\circ} 4,2009$, pp. 1-14

- SÁNCHEZ MARCO,A. (2010) New data and an overview of the past avifaunas from the Canary Islands. Ardeola, vol. 57, n. $^{\circ} 1,2010$, pp. $13-40$

- SÁNCHEZ MARCO, A. (2015) Aves del Pleistoceno inferior de Cueva Victoria (costa sudoriental mediterránea de la península Ibérica). En GIBERT, L.; FERRÀNDEZCAÑADELL, C. (ed.) Geología y Paleontología de Cueva Victoria. Mastia, vol. 11/13, 2015, pp. 253-267 
- SÁNCHEZ MARCO, A. (2017) Las rapaces y otras aves de Batallones: 383-391. En MORALES, J. (ed.) La colina de los tigres dientes de sable. Los yacimientos miocenos del cerro de los Batallones. Comunidad de Madrid: Museo Arqueológico Regional, 2017

- SÁNCHEZ MARCO, A. (2018) Las aves de la excavación histórica de El Castillo: 280-300. En CASTAÑOS, P. M. (ed.) El Castillo: historia de una fauna olvidada. Cantabria: Gobierno de Cantabria, Consejería de Educación Cultura y Deporte, 2018 (Monografías del Museo de Prehistoria y Arqueología de Cantabria, 1)

- SÁNCHEZ MARCO, A. (en prensa) Miocene birds from the Valles-Penedes basin. Chapter 4. En ALBA, D. M.; SOLÀ-MOYÀ, S.; ALMÉCIJA, S. (ed.) Fossil hominoid primates from the Valles-Penedes basin: taxonomy and paleobiology. New York: Springer Publishers (Vertebrate Paleobiology and Paleoanthropology Book Series)

- SANZ, J. L.; CHIAPPE, L. M.; PÉREZ-MORENO, B. P.; MORATALLA, J. J.; HERNÁNDEZ-CARRASQUILLA, F.; BUSCALIONI, A. D.; ORTEGA, F.; POYATO-ARIZA, F. J.; RASSKIN-GUTMAN, D.; MARTÍNEZ-DELCLÒS, X. (1997) A nestling bird from the Lower Cretaceous of Spain: implications for avian skull and neck evolution. Science, 276, 1997, pp. 1543-1546

- SANZ, J. L.; ORTEGA, F. (2002) The birds from Las Hoyas. Science Progress, vol. 85, n. ${ }^{\circ} 2,2002$, pp. 113-130

- STEAdMAN, D. W. (1989) Extinction of birds in eastern Polynesia: a review of the record, and comparisons with other Pacific island groups. Journal of Archaeological Science, vol. 16, 1989, pp. 177-205

- STEADMAN, D. W. (2006) Extinction and biogeography of Tropical Pacific birds. Chicago: Chicago University Press

- STEADMAN, D. W.; ROLETT, B. (1996) A chronostratigraphic analysis of landbird extinction on Tahuata, Marquesas islands. Journal of Archaeological Science, vol. 23, 1996, pp. 81-94

- WALKER, C. A.; WRAGG, G. M.; HARRISON, C. J. O. (1990) A new shearwater from the Pleistocene of the Canary Islands and its bearing on the evolution of certain Puffinus shearwaters. Historical Biology, vol. 3, 1990, pp. 203-224

- WANG, S., HU, Y.; WANG, L. (2011) New ratite eggshell material from the Miocene of Inner Mongolia, China. Chinese Birds, vol. 2, 2011, n. ${ }^{\circ}$ 1, pp. 28-26

- ZELENKOV, N.V.; MANEGOLD, A. (2015) A new species of Aegypius vulture from the early Pliocene of Moldova is the earliest unequivocal evidence of Aegypiinae in Europe. Paläontol. Z., vol. 89, 2015, pp. 529-534

- ZHANG, Z; ZHENG, X.; ZHENG, G. (2010) A new Old World vulture (Falconiformes: Accipitridae) from the
Miocene of Gansu province, northeast China. Journal of Ornithology, vol. 151, 2010, pp. 401-408

- ZHOU, Z. (2004) The origin and early evolution of birds: discoveries, disputes and perspectives from fossil evidence. Naturwissenschaften, vol. 91, 2004, pp. 455-471 